\title{
ACOUSTIC RADIATION FROM A SEMI-INFINITE ANNULAR DUCT IN A UNIFORM SUBSONIC MEAN FLOW
}

\author{
S. W. RIENSTRA \\ National Aerospace Laboratory NLR, Amsterdam, The Netherlands
}

(Received 12 January 1983, and in revised form 2 August 1983)

\begin{abstract}
By using a Wiener-Hopf approach, an analytical description is derived of the scattered field of a harmonic sound wave coming out of an open ended annular duct (a semi-infinite cylinder inside of which, coaxially, is a doubly infinite hub), submerged in a subsonic, coaxial, uniform mean flow. The possibility of vortex shedding from the pipe exit edge is included. Explicit expressions are given for the acoustic power inside the pipe, in the acoustic far field and, in the presence of vortex shedding, in the hydrodynamic far field, and of the power absorbed by the vortex sheet. The formulae are evaluated numerically with the aid of asymptotic expansions, and a method in which complex contour deformation is used, which is more convenient than those usually employed for this type of diffraction problem. The equality of power appeared to be an important check on the calculations. A numerical survey is made of the behaviour of the acoustic power loss, due to vortex shedding from the trailing edge, at frequencies near cut-off, as a function of Mach number, mode number of the incident wave, and hub radius. The power loss appears to increase with increasing Mach number, increasing hub radius and with decreasing frequency. Only in the case of the plane wave (where $k \rightarrow 0$ ) does the ratio of radiated and transmitted power become zero; for the other modes (at their cut-off frequencies) this ratio tends to a finite value. Somewhat surprising is that, in comparison with the jet, the power loss in a uniform flow is much higher. As a typical example for higher frequencies, the far field radiation pattern of a $k=50, m=4$ wave is considered as a function of the Kutta condition and hub radius.
\end{abstract}

\section{INTRODUCTION}

Sound propagation in cylindrical, infinite ducts is a classical problem of acoustics, which has for long been solved mathematically as an eigenvalue problem (see, e.g., the book by Rayleigh [1], section 18.340). The related problem of reflection at and radiation from an open end (without flange) is much more difficult, and has been solved with the aid of the Wiener-Hopf technique, most appropriate for this type of half-range diffraction problems, by Levine and Schwinger [2]. For the aeronautical application of a jet engine, the problem of sound radiation from a semi-infinite pipe in a uniform subsonic flow, coaxial with the pipe, is of interest. If the medium flows into the pipe, the acoustic field is continuous everywhere, and, after a Lorentz-transformation, one can effectively use the no-flow solution, but if the flow is directed out of the pipe, the acoustic field sheds from the trailing edge a vortex sheet, which has to be included in the solution. This was shown by Carrier [3], and elaborated by Munt [4], who also showed the significance of the vortex sheet for the acoustic far field. Although vorticity is quiet on its own, it induces, when moving near a solid body, pressure variations on this body, which develop as sound radiating away [5]. To include a jet engine inlet of annular shape, Friedrich [6] considered the problem of sound reflection and radiation from an annular duct (a semi-infinite pipe 
inside of which is a doubly infinite hub) with uniform inlet flow, thus without considering the shedding of vorticity.

In what follows here these analyses are combined to provide a comprehensive description of the sound field within and radiating out of an annular duct, including the limit of the hub diameter tending to zero, with a uniform subsonic mean flow, into or out of the pipe, and with a variable edge condition (i.e., the rate of vortex shedding is a free parameter). Particular attention is given to the acoustic energy balance, and its relation to the hydrodynamic field of the shed vorticity. In addition, a method is described, based on complex contour deformation, for evaluating the analytical formulae numerically more easily than has usually been presented in the literature. This method is particularly profitable at high frequencies.

A further improvement to model an aero-engine exhaust flow (which is not developed here) is a uniform jet separated from an ambient flow by a stationary (non-acoustic) vortex sheet [7]. Here also the scattering acoustic wave sheds vorticity from the edge (additional to the mean flow vortex sheet), but now triggering a Helmholtz instability of the mean flow. Another step along these lines would be a semi-infinite hub, possibly from which is issuing an inner jet. This problem can be written as a generalized Wiener-Hopf equation, but is much more difficult to solve than the one considered here.

\section{FORMULATION OF THE PROBLEM}

An inviscid fluid with density $\rho_{0}^{*}$, pressure $p_{0}^{*}$ and sound speed $c_{0}^{*}$ flows in $\left(x^{*}, r^{*}, \theta\right)$ space with uniform subsonic velocity $U_{0}^{*}$, in the positive $x^{*}$ direction if $U_{0}^{*}$ is positive, along a semi-infinite pipe, located at $x^{*}<0$ and $r^{*}=b^{*}$, inside of which is a doubly infinite hub, located at $r^{*}=h^{*}, 0 \leqslant h^{*}<b^{*}$ (see Figure 1). The flow is perturbed by sound,

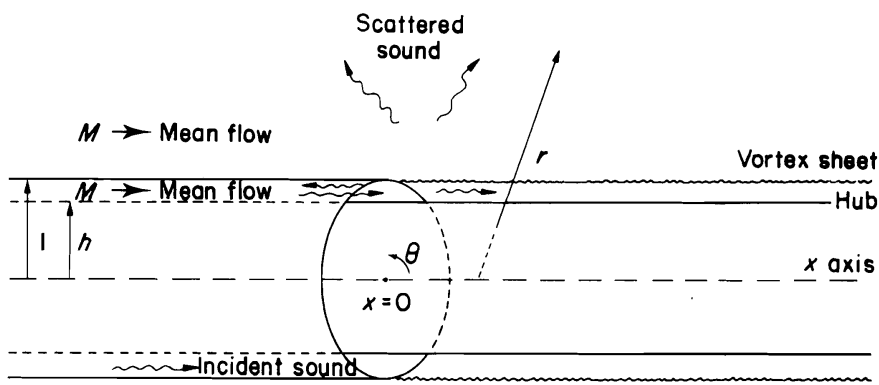

Figure 1. Sketch of the geometry.

coming from inside the pipe. For convenience, the incident wave is assumed to consist of one (so-called) $(m, \mu)$-pipe mode (see below), varying harmonically in time with frequency $f^{*}>0$; any incident sound field can be built up then by a Fourier-Bessel superposition. The perturbation amplitudes are considered to be small enough for linearization. Apart from the pipe and hub surface and the possible wake behind the pipe edge, there are no regions containing vortical flow, and one can introduce a potential $\phi^{*}$ for the velocity perturbations $\vec{\nabla}^{*} \phi^{*}$. The variables are made dimensionless as follows: the space co-ordinates $x^{*}=x b^{*}, r^{*}=r b^{*}$; the time co-ordinate $t^{*}=t b^{*} / c_{0}^{*}$; the velocities $\vec{\nabla}^{*} \phi^{*}=\vec{\nabla} \phi c_{0}^{*}$; the density $\rho^{*}=(1+\rho) \rho_{0}^{*}$; the pressure $p^{*}=p_{0}^{*}+\rho_{0}^{*} c_{0}^{* 2} p$. The dimensionless radian frequency, or Helmholtz number, is $k=2 \pi f^{*} b^{*} / c_{0}^{*}(k>0)$, and the main flow Mach number is $M=U_{0}^{*} / c_{0}^{*}(-1<M<1)$. 
The equations and boundary conditions for this model are as follows:

$$
\begin{gathered}
p=\rho \quad(\text { acoustic approximation); } \\
p=-(\partial / \partial t+M \partial / \partial x) \phi \quad \text { (Bernoulli's equation, linearized); } \\
(\partial / \partial t+M \partial / \partial x) \rho+\nabla^{2} \phi=0 \quad \text { (continuity equation, linearized); } \\
\partial \phi / \partial r=0 \quad \text { if } r=h, \text { or } r=1 \text { and } x<0 \quad \text { (rigid walls); }
\end{gathered}
$$$$
p \text { continuous across } r=1, x>0 ; \quad p-p_{\text {in }} \text { radiating outwards at infinity. }
$$

The incident, primary wave $p_{\text {in }}$ is taken as a wave form of a doubly infinite pipe which retains its shape as it travels down the pipe (i.e., shows no dispersion), and is described by

$$
p_{\text {in }}(x, r, \theta, t)=\left\{\begin{array}{ll}
U_{m \mu}(r) \exp \left(-\mathrm{i} k_{m \mu}^{+} x+\mathrm{i} m \theta+\mathrm{i} k t\right), & h<r<1 \\
0, & 1<r<\infty
\end{array}\right\},
$$

where $U_{m \mu}$ is a linear combination of $m$ th order Bessel functions of the first kind $\left(\mathrm{J}_{m}\right)$ and of the second kind $\left(\mathrm{Y}_{m}\right)[8]$,

$$
\begin{gathered}
U_{m \mu}(r)=N_{m \mu} \cos \tau_{m \mu} \mathrm{J}_{m}\left(\alpha_{m \mu} r\right)-N_{m \mu} \sin \tau_{m \mu} \mathrm{Y}_{m}\left(\alpha_{m \mu} r\right) \quad(m \geqslant 0, \mu \geqslant 1), \\
N_{m \mu}=\frac{1}{2} \sqrt{2} \pi \alpha_{m \mu}\left[\frac{1-m^{2} / \alpha_{m \mu}^{2}}{\mathrm{~J}_{m}^{\prime}\left(\alpha_{m \mu}\right)^{2}+\mathrm{Y}_{m}^{\prime}\left(\alpha_{m \mu}\right)^{2}}-\frac{1-m^{2} / \alpha_{m \mu}^{2} h^{2}}{\mathrm{~J}_{m}^{\prime}\left(\alpha_{m \mu} h\right)^{2}+\mathrm{Y}_{m}^{\prime}\left(\alpha_{m \mu} h\right)^{2}}\right]^{-1 / 2} \\
\tau_{m \mu}=\arctan \left[J_{m}^{\prime}\left(\alpha_{m \mu}\right) / \mathrm{Y}_{m}^{\prime}\left(\alpha_{m \mu}\right)\right]
\end{gathered}
$$

in which $\alpha_{m \mu}$ is the $\mu$ th positive zero of $\mathrm{J}_{m}^{\prime}(\alpha) \mathrm{Y}_{m}^{\prime}(\alpha h)-\mathrm{Y}_{m}^{\prime}(\alpha) \mathrm{J}_{m}^{\prime}(\alpha h)$, including $\alpha_{01}=0$, where the sequence $\left\{\alpha_{m \mu}\right\}$ is infinite and real. (This is an immediate consequence of the Sturm-Liouville theory for eigenvalue problems [6].) The corresponding axial wave numbers are defined by $k_{m \mu}^{+}=\left(\Omega_{m \mu}-M k\right) / \beta^{2}$, where $\beta^{2}=1-M^{2}$ and in which $\Omega_{m \mu}=$ $\left(k^{2}-\beta^{2} \alpha_{m \mu}^{2}\right)^{1 / 2}$ if $k>\beta \alpha_{m \mu}$ and $\Omega_{m \mu}=-\mathrm{i}\left(\beta^{2} \alpha_{m \mu}^{2}-k^{2}\right)^{1 / 2}$ otherwise. If $m=0$, the first non-trivial solution is the plane wave, corresponding to $\alpha_{01}=0$, and $U_{01}(r)=N_{01}=$ $\sqrt{2} / \sqrt{1-h^{2}}$. Furthermore, if $h=0$, the definition of $U_{m \mu}$ simply degenerates to its limiting value, given by $U_{m \mu}(r)=N_{m \mu} \mathrm{J}_{m}\left(\alpha_{m \mu} r\right), \quad N_{m \mu}=\left\{\frac{1}{2}\left(1-m^{2} / \alpha_{m \mu}^{2}\right) \mathrm{J}_{m}\left(\alpha_{m \mu}\right)^{2}\right\}^{-1 / 2}$ and $\mathrm{J}_{m}^{\prime}\left(\alpha_{m \mu}\right)=0$, while $U_{01}=\sqrt{ } 2$.

Mathematically, these waveforms, or modes, are to be identified with eigensolutions of the prevailing infinite pipe equations, and the sequences $\{m\}$ and $\left\{\alpha_{m \mu}\right\}$ with the corresponding eigenvalues. In view of the symmetry, only positive values of $m$ and $\alpha_{m \mu}$ are to be considered here. With these eigensolutions any solution can be constructed, provided positive and negative values of $m$ and $\Omega_{m \mu}$ (for counter rotating and backward running modes) are included.

The system (2.1) with boundary conditions does not have a unique solution, since it allows an axial velocity $\partial \phi / \partial x$ to be discontinuous along $r=1, x>0$, the extension of the pipe. This discontinuity corresponds to a distribution of vorticity, shed from the pipe edge, and is directly related to the velocity singularity at the edge. The existence of a vortex layer is normal in case of a trailing edge $(M>0)$, but is physically unrealistic for a leading edge (intake) configuration with $M<0$. As a measure of the amount of shed vorticity, a complex parameter $\gamma$ can be introduced. This parameter is to be taken equal to zero if no vortex shedding is permitted ( $\phi$ continuous), and equal to unity if so much vorticity is to be shed that the velocity singularity at the edge $x=0, r=1$ is removed (Kutta condition). If required, any other (complex) value of $\gamma$ is possible. 
Since the scattered field must have the same behaviour in $t$ and in $\theta$ as its cause $p_{\text {in }}$, one can write $\phi(x, r, \theta, t)=\phi(x, r) \exp (\mathrm{i} m \theta+\mathrm{i} k t)$ (analogously for the other variables). Then the equations (2.1) can be combined to yield the following equation for $\psi=$ $\phi-\mathrm{i}\left(k-M k_{m \mu}^{+}\right)^{-1} p_{\text {in }}$, the potential of the scattered field:

$$
\left[\partial^{2} / \partial r^{2}+(1 / r) \partial / \partial r-\left(m^{2} / r^{2}\right)+\partial^{2} / \partial x^{2}-(\mathrm{i} k+M \partial / \partial x)^{2}\right] \psi=0
$$

with boundary conditions $\partial \psi / \partial r=0$ if $r=h$, or $r=1$ and $x<0, p_{\text {in }}-(\mathrm{i} k+M \partial / \partial x) \psi$ continuous across $r=1, x>0$, and $\psi$ radiating outwards at infinity. The condition of " $\psi$ radiating outwards at infinity" presents some conceptual difficulties for $r=1$ and $x \rightarrow \infty$ in the presence of a vortex layer. "Radiating" can be interpreted as relating to the acoustic energy flux vector, a quantity which is undefined for an acoustic field in a mean flow with vorticity [9, p. 41]. However, since the sound field can be expected to show no singular behaviour along $r=1, x>0$, and the vorticity to generate no sound at infinity but to be (for $M>0$ ) convected away with the main stream, there seem to be no practical problems.

The equation (2.3) with boundary conditions will be the problem to be solved in the next section. The method employed will be Fourier transforms on $x$ of appropriate dependent variables, together with application of the Wiener-Hopf technique [10].

\section{ANALYTICAL SOLUTION}

One can introduce (formally) the Fourier transforms

$$
\begin{gathered}
\Psi(u, r)=\int_{-\infty}^{\infty} \psi(x, r) \mathrm{e}^{\mathrm{i} k u x} \mathrm{~d} x, \quad F_{+}(u)=\int_{0}^{\infty} \frac{\partial}{\partial r} \psi(x, 1) \mathrm{e}^{\mathrm{i} k u x} \mathrm{~d} x, \\
P_{-}(u)=\int_{-\infty}^{0}\left(-\mathrm{i} k-M \frac{\partial}{\partial x}\right)[\psi(x, 1+)-\psi(x, 1-)] \mathrm{e}^{\mathrm{i} k u x} \mathrm{~d} x,
\end{gathered}
$$

where $1+$ and $1-$ mean upper and lower limit, respectively. Physically, $F_{+}$denotes the transform of the total time derivative of the position of the dividing streamline, emanating from the edge, and $P_{-}$denotes the pressure difference across the pipe wall as induced by the $\psi$-field. It is convenient to write $k$ with a small imaginary part, $k=|k| \mathrm{e}^{-\mathrm{i} \delta}, 0<\delta \ll 1$, and, at the end of the analysis, to set $\delta$ to zero. Also it is convenient to introduce the square roots $w_{+}(u)=[1-(1+M) u]^{1 / 2}$ and $w_{-}(u)=[1+(1-M) u]^{1 / 2}$, both defined as principal branches, and yielding $w(u)=w_{+}(u) w_{-}(u)$.

To set up a Wiener-Hopf equation one assumes that $\psi$ is sufficiently well behaved for the transforms (3.1) to exist in a strip $S$, defined as the intersection of the halfplanes $R_{+}$ and $R_{-}$(see Figure 2):

$$
\begin{aligned}
& R_{+}=\left\{u \in \mathbb{C} \mid \operatorname{Im} u>\tan \delta\left(\operatorname{Re} u+M \beta^{-2}-\sigma\right)\right\} \\
& R_{-}=\left\{u \in \mathbb{C} \mid \operatorname{Im} u<\tan \delta\left(\operatorname{Re} u+M \beta^{-2}+\sigma\right)\right\}
\end{aligned}
$$

The constant $\sigma$ is real, positive, and sufficiently small to leave out of $S$ the branch cuts of $w_{+}$and $w_{-}$, and certain poles to be discussed later. A function analytic in $R_{+}$will be denoted by an index + , and a function analytic in $R_{-}$by an index - . In view of their half range integration intervals, $F_{+}$and $P_{-}$are anticipated to be indeed analytic in $R_{+}$and $R_{-}$, respectively $[10$, p. X]. With the introduction of $\Psi$, equation (2.3) reduces to

$$
\left[\mathrm{d}^{2} / \mathrm{d} r^{2}+(1 / r) \mathrm{d} / \mathrm{d} r+k^{2} w^{2}-m^{2} / r^{2}\right] \Psi=0 \quad(u \in S),
$$




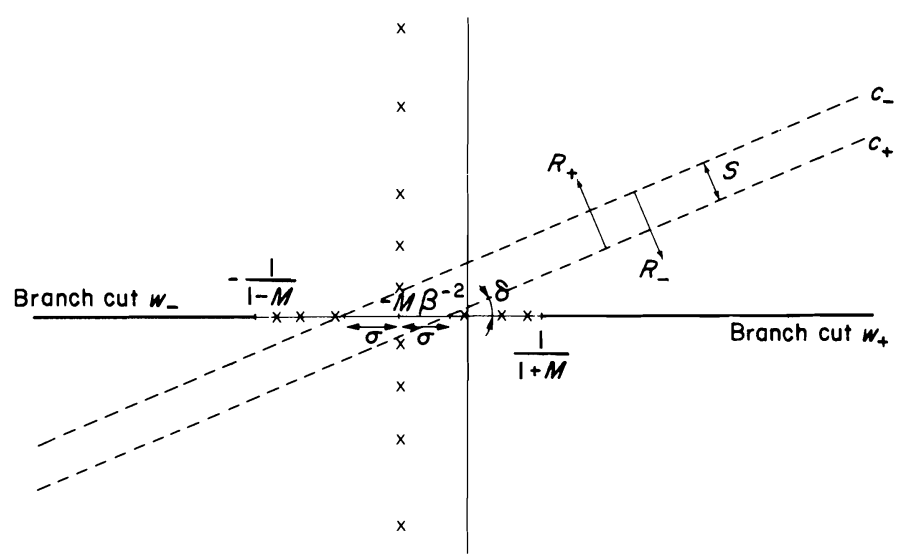

Figure 2. The complex $u$ plane, with branch cuts of $w_{+}$and $w_{-}$, subdomains $R_{+}, R_{-}$and $S$, zeros $k_{m \mu}^{+}$and $k_{m_{\mu}}^{-}(\times)$, and integration contours $c_{+}$and $c_{-}$.

with solution, for $h<r<1$,

$$
\Psi(u, r)=A(u)\left\{\mathrm{Y}_{m}^{\prime}(k w h) \mathrm{J}_{m}(k w r)-\mathrm{J}_{m}^{\prime}(k w h) \mathrm{Y}_{m}(k w r)\right\}
$$

for the hard-wall condition at $r=h$, and for $1<r<\infty$,

$$
\Psi(u, r)=B(u) \mathrm{H}_{m}^{(2)}(k w r) .
$$

Here the fact that $\operatorname{Re} w(u)>0$ if $u \in S$ together with the radiation condition has been used, implying that $\Psi$ tends to zero for $r \rightarrow \infty, \mathrm{H}_{m}^{(2)}=\mathrm{J}_{m}-\mathrm{iY} \mathrm{Y}_{m}$ denotes the $m$ th order Hankel function of the second kind [8].

From continuity of pressure along $r=1, x>0$, it follows that

$$
-\mathrm{i} k(1-M u)[\Psi(u, 1+)-\Psi(u, 1-)]=P_{-}(u)+\mathrm{i} U_{m \mu}(1) / k\left(u-k_{m \mu}^{+} / k\right),
$$

and from the hard wall condition along $r=1, x<0$, that $(\mathrm{d} / \mathrm{d} r) \Psi(u, 1)=F_{+}(u)$. Then

$$
\begin{gathered}
A(u)=F_{+}(u) /\left\{\mathrm{Y}_{m}^{\prime}(k w h) \mathrm{J}_{m}^{\prime}(k w)-\mathrm{J}_{m}^{\prime}(k w h) \mathrm{Y}_{m}^{\prime}(k w)\right\} k w, \\
B(u)=F_{+}(u) / \mathrm{H}_{m}^{(2) \prime}(k w) k w, \\
-2 \mathrm{i}(1-u M) F_{+}(u) / k w^{2}(u) K(u)=P_{-}(u)+\mathrm{i} U_{m \mu}(1) / k\left(u-k_{m \mu}^{+} / k\right), \\
K(u)=L(k w(u))=\tilde{K}(\tau)=L(k v(\tau) / \beta), \\
L(z)=\pi\left[\mathrm{H}_{m}^{(2) \prime}(z) / \mathrm{H}_{m}^{(2) \prime}(z h)\right]\left\{\mathrm{J}_{m}^{\prime}(z h) \mathrm{Y}_{m}^{\prime}(z)-\mathrm{Y}_{m}^{\prime}(z h) \mathrm{J}_{m}^{\prime}(z)\right\}, \\
\tau=\beta^{2} u+M, \quad v(\tau)=v_{+}(\tau) v_{-}(\tau)=(1-\tau)^{1 / 2}(1+\tau)^{1 / 2} .
\end{gathered}
$$

The square roots $v_{+}$and $v_{-}$are principal branches, and $\alpha_{m \nu}$ is defined as the $\nu$ th $(\nu \geqslant 1)$ zero of $\mathrm{J}_{m}^{\prime}(z h) \mathrm{Y}_{m}^{\prime}(z)-\mathrm{Y}_{m}^{\prime}(z h) \mathrm{J}_{m}^{\prime}(z)$. It is now convenient to introduce the notation

$$
\begin{gathered}
k_{m \nu}^{ \pm}=k \bar{k}_{m \nu}^{ \pm}=k\left( \pm \bar{\Omega}_{m \nu}-M\right) / \beta^{2} \\
\bar{\Omega}_{m \nu}=\left\{\begin{array}{ll}
\left\{1-\left(\beta \alpha_{m \nu} / k\right)^{2}\right\}^{1 / 2} & \text { if } k>\beta \alpha_{m \nu} \\
-i\left\{\left(\beta \alpha_{m \nu} / k\right)^{2}-1\right\}^{1 / 2} & \text { otherwise }
\end{array}\right\}, \quad \Omega_{m \nu}=k \bar{\Omega}_{m \nu}
\end{gathered}
$$


Evidently, if $\bar{\Omega}_{m \nu} \neq 0$ for all $\nu$ (no resonance), and $\sigma$ is sufficiently small the sequence $\left\{k_{m \nu}^{+}\right\}$forms a set of zeros of $K(u)$ in $R_{-} \mid S$, and $\left\{k_{m \nu}^{-}\right\}$a set of zeros of $K(u)$ in $R_{+} \mid S$. One can now state (without proof) that, in view of the location of zeros and poles of $\mathrm{H}_{m}^{(2) \prime}(z) / \mathrm{H}_{m}^{(2) \prime}(z h)$, it is possible to take $\sigma$ small enough for $K(u)$ to be analytic and non-zero in $S$. Then it is routine to define the split functions $K_{+}$and $K_{-}$, where $K(u)=$ $K_{+}(u) K_{-}(u)$ and $K_{ \pm}$are analytic and non-zero in $R_{ \pm}$, by

$$
\log K_{ \pm}(u)= \pm \frac{1}{2 \pi \mathrm{i}} \int_{c_{ \pm}} \frac{\log K\left(u^{\prime}\right)}{u^{\prime}-u} \mathrm{~d} u^{\prime}
$$

for $u \in R_{ \pm}$. The contours $c_{ \pm}$(Figure 2) are defined by $\operatorname{Im} u^{\prime}=\tan \delta\left(\operatorname{Re} u^{\prime}+M \beta^{-2} \mp \sigma\right)$. The integrals of $K_{ \pm}$have to be treated with some care (cf. [10, p. 42]) since they are not normally convergent, but later they are to be transformed into a convergent form, appropriate for numerical evaluation, so it is not necessary to discuss this point further here. One can now rewrite equation (3.2) into one with on the left-hand side only + functions and at the right-hand side only - functions, as follows:

$$
\begin{aligned}
& 2(1-u M) F_{+}(u) / w_{+}^{2}(u) K_{+}(u)+U_{m \mu}(1) w_{-}^{2}\left(\bar{k}_{m \mu}^{+}\right) K_{-}\left(\bar{k}_{m \mu}^{+}\right) /\left(u-\bar{k}_{m \mu}^{+}\right) \\
& \quad=\mathrm{i} k w_{-}^{2}(u) K_{-}(u) P_{-}(u)-U_{m \mu(1)}\left[w_{-}^{2}(u) K_{-}(u)-w_{-}^{2}\left(\bar{k}_{m \mu}^{+}\right) K_{-}\left(\bar{k}_{m \mu}^{+}\right)\right] /\left(u-\bar{k}_{m \mu}^{+}\right) .
\end{aligned}
$$

From the identity theorem for analytic functions this equation defines one entire function $E(u)$. Since $K(u)=K\left(-u-2 M / \beta^{2}\right)$ and $K(u)=L(k w(u)) \cong L(-\mathrm{i} k \beta|u|) \cong 1 / k \beta|u|$ for $|u| \rightarrow \infty$ in $S$, it follows, in a way analogous to that in the book by Noble [10, p. 42], that $K_{ \pm}(u)=O\left(u^{\mp 1 / 2}\right)$ for $|u| \rightarrow \infty$ in $R_{ \pm}$. Continuity of the streamline emanating from the edge, and the assumption, a priori (to be verified a posteriori), of algebraic behaviour of $\psi$ near the edge, requires $(\partial / \partial r) \psi(x, 1)$ to behave like $x^{\eta-1}(x \downarrow 0)$ with $\eta>0$, which, in turn, implies, by using a variant of the theorem as given by Noble [10, p. 36], that $F_{+}(u)=O\left(u^{-\eta}\right)$ for $u \rightarrow \infty$ in $R_{+}$. Then, if one assumes $P_{-}(u)$ to behave algebraically for $u \rightarrow \infty$ in $R_{-}$(again, a priori and to be verified afterwards), it appears from the behaviour at infinity of $E(u)$, by using the extended form of Liouville's theorem [10, p. 6], that $E$ must be a constant, and $\eta=\frac{1}{2}$. In addition, going one step further, in the case of the Kutta condition $F_{+}(u)=O\left(u^{-3 / 2}\right)\left(u \rightarrow \infty\right.$ in $\left.R_{+}\right)$and $E=0$. Now, upon recognizing the pole $u=M^{-1}$ in $F_{+}$to represent mathematically the vortex shedding, it is natural to write the constant $E$ as dependent on a parameter $\gamma$ (as anticipated in the previous section) in a way such that $\gamma=0$ corresponds to no vortex shedding (cancellation of the pole $u=M^{-1}$ ), and $\gamma=1$ to the Kutta condition $(E=0)$. This is achieved if one takes

$$
E=(1-\gamma) U_{m \mu}(1) w_{-}^{2}\left(\bar{k}_{m \mu}^{+}\right) K_{-}\left(\bar{k}_{m \mu}^{+}\right) /\left(M^{-1}-\bar{k}_{m \mu}^{+}\right) \text {. }
$$

Then

$$
F_{+}(u)=-\frac{1}{2} \frac{U_{m \mu}(1) w_{-}^{2}\left(\bar{k}_{m \mu}^{+}\right) K_{-}\left(\bar{k}_{m \mu}^{+}\right)}{1-M \bar{k}_{m \mu}^{+}} \frac{w_{+}^{2}(u) K_{+}(u)}{1-u M}\left[\frac{1-u M}{u-\bar{k}_{m \mu}^{+}}+M \gamma\right] .
$$

With substitution of $F_{+}$into the expressions for $\Psi$ and with $\delta \rightarrow 0$, the problem of finding an analytical expression for $\psi$ is now, at least in principle, solved. Further simplification of the formulae (which are, being double integrals, complicated) is possible for points of observation inside the pipe, and in the far field outside the pipe. Being of great practical interest, these two cases will be evaluated in the next two sections.

Finally, it can be noted that the case $h=0$ follows immediately from the above formulae if one takes the limit $h \downarrow 0$. Then

$$
L(z)=-\pi \mathrm{iH}_{m}^{(2) \prime}(z) \mathrm{J}_{m}^{\prime}(z), \quad \Psi(u, r<1)=F_{+}(u) \mathrm{J}_{m}(k w r) / k w \mathrm{~J}_{m}^{\prime}(k w) .
$$




\section{THE FIELD INSIDE THE PIPE}

From the inverse transform of $\Psi$ the pressure field for $h<r<1$ is

$$
\begin{gathered}
p(x, r)=p_{\mathrm{in}}(x, r)+\frac{k^{2}}{2 \pi \mathrm{i}} \int_{-\infty-\mathrm{i} 0}^{\infty+\mathrm{i} 0}(1-u M) \Psi(u, r) \mathrm{e}^{-\mathrm{i} k u x} \mathrm{~d} u \\
\Psi(u, r)=F_{+}(u) \frac{1}{k w} \frac{\mathrm{Y}_{m}^{\prime}(k w h) \mathrm{J}_{m}(k w r)-\mathrm{J}_{m}^{\prime}(k w h) \mathrm{Y}_{m}(k w r)}{\mathrm{Y}_{m}^{\prime}(k w h) \mathrm{J}_{m}^{\prime}(k w)-\mathrm{J}_{m}^{\prime}(k w h) \mathrm{Y}_{m}^{\prime}(k w)} .
\end{gathered}
$$

In view of the exponential behaviour and the presence of only poles of the integrand in the upper half plane, it is profitable, if $x<0$, to close the contour via a large semi-circle in the upper half plane, and write the field as a sum of residue contributions, the so-called modal representation. This requires a little study of the function

$$
f_{m}(z)=\frac{1}{z} \frac{\mathrm{Y}_{m}^{\prime}(z h) \mathrm{J}_{m}(z r)-\mathrm{J}_{m}^{\prime}(z h) \mathrm{Y}_{m}(z r)}{\mathrm{Y}_{m}^{\prime}(z h) \mathrm{J}_{m}^{\prime}(z)-\mathrm{J}_{m}^{\prime}(z h) \mathrm{Y}_{m}^{\prime}(z)}
$$

Substitution into this expression of the series defining $\mathbf{J}_{m}$ and $Y_{m}$ reveals that $f_{m}(z)$ is a meromorphic function (analytic everywhere except for isolated poles) of $z^{2}$, implying that $f_{m}(k w(u))$ is meromorphic in $u$. Furthermore, it shows that $f_{m}(z)=O(1)(z \rightarrow 0)$ if $m \neq 0$, while $f_{0}(z)=O\left(z^{-2}\right)(z \rightarrow 0)$. The residues of interest stem from the poles at $u=\bar{k}_{m \nu}^{-}$, which are related to the poles $z=\alpha_{m \nu}$ of $f_{m}(z)$. Using the identities

$$
\mathrm{J}_{m}^{\prime}\left(\alpha_{m \nu}\right) / \mathrm{J}_{m}^{\prime}\left(\alpha_{m \nu} h\right)=\mathrm{Y}_{m}^{\prime}\left(\alpha_{m \nu}\right) / \mathrm{Y}_{m}^{\prime}\left(\alpha_{m \nu} h\right)=h U_{m \nu}(h) / U_{m \nu}(1)
$$

one can derive

$$
\begin{gathered}
\lim _{z \rightarrow \alpha_{m \nu}}\left(z-\alpha_{m \nu}\right) f_{m}(z)=-\left(1 / 2 \alpha_{m \nu}\right) U_{m \nu}(1) U_{m \nu}(r) \quad(m \geqslant 1, \nu \geqslant 1, \text { or } m=0, \nu \geqslant 2), \\
\lim _{z \rightarrow 0} z^{2} f_{0}(z)=-2 /\left(1-h^{2}\right) \quad(m=0, \nu=1) .
\end{gathered}
$$

Hence

$$
\begin{gathered}
\lim _{u \rightarrow \bar{k}_{m \nu}^{-}}\left(u-\bar{k}_{m \nu}^{-}\right) f_{m}(k w(u))=-\left(1 / 2 k^{2} \bar{\Omega}_{m \nu}\right) U_{m \nu}(1) U_{m \nu}(r), \\
\lim _{u \rightarrow \bar{k}_{01}^{-}}\left(u-\bar{k}_{01}^{-}\right) f_{0}(k w(u))=-1 / k^{2}\left(1-h^{2}\right)
\end{gathered}
$$

Since $\bar{\Omega}_{01}=1$, one can see that for the case $m=0, \nu=1$, although the treatment is different, the final result is consistent with the general result. Now one is able to describe the field inside the pipe. In a quite natural way the so-called modal form appears, of an incident wave scattered into backward running waves of the same $m$-mode number, with amplitudes $R_{m \mu \nu}$ times the primary wave amplitude. $\left[R_{m \mu \nu}\right]$ is called the reflection coefficient matrix. Thus

$$
\begin{aligned}
p(x, r)=U_{m \mu}(r) & \exp \left(-\mathrm{i} k_{m \mu}^{+} x\right)+\sum_{\nu=1}^{\infty} R_{m \mu \nu} U_{m \nu}(r) \exp \left(-i k_{m \nu}^{-} x\right), \\
R_{m \mu \nu}= & -\frac{1}{4} U_{m \mu}(1) U_{m \nu}(1) \frac{\left(1+\bar{\Omega}_{m \mu}\right)\left(1+\bar{\Omega}_{m \nu}\right)}{\bar{\Omega}_{m \nu}\left(\bar{\Omega}_{m \mu}+\bar{\Omega}_{m \nu}\right)} \\
& \times \frac{1-M \gamma \bar{\Omega}_{m \mu}-M(\gamma-1) \bar{\Omega}_{m \nu}}{1-M \bar{\Omega}_{m \mu}}\left(\bar{\Omega}_{m \mu}\right) \tilde{K}_{-}\left(\bar{\Omega}_{m \nu}\right) .
\end{aligned}
$$


The way $\tilde{K}_{ \pm}$is defined is analogous to that for $K_{ \pm}$. Hence

$$
K_{ \pm}\left(\bar{k}_{m \nu}^{\mp}\right)=\exp \left[ \pm \frac{1}{2 \pi \mathrm{i}} \int_{-\infty-\mathrm{i} 0}^{\infty+\mathrm{i} 0} \frac{\log \tilde{K}(\tau)}{\tau \pm \bar{\Omega}_{m \nu}} \mathrm{d} \tau\right]=\tilde{K}_{ \pm}\left(\mp \bar{\Omega}_{m \nu}\right)
$$

the contour of integration crossing the real axis at $\tau=0$. Due to the symmetry of $\tilde{K}(\tau)$ in $\tau$, it follows that $\tilde{K}_{+}\left(-\bar{\Omega}_{m \nu}\right)=\tilde{K}_{-}\left(\bar{\Omega}_{m \nu}\right)$. This expression for $R_{m \mu \nu}$ is equally valid for $h=0$, provided, of course, that the appropriate limiting forms for $U_{m \mu}$ and $\tilde{K}$, as mentioned before, are taken.

To investigate the behaviour of the reflection matrix near cut-off frequencies one has to distinguish between various cases and combinations of parameters. First, if $k \rightarrow \alpha_{m \mu} \beta \neq 0$ (i.e., $\bar{\Omega}_{m \mu} \rightarrow 0$ ),

$$
\tilde{K}_{-}\left(\bar{\Omega}_{m \mu}\right) \cong \exp \left[\frac{\mathrm{i} \bar{\Omega}_{m \mu}}{\pi} \oint_{0}^{\infty} \frac{\log \left[L\left(\alpha_{m \mu}\right)+\frac{1}{2} \alpha_{m \mu}\left(\bar{\Omega}_{m \mu}^{2}-\tau^{2}\right) L^{\prime}\left(\alpha_{m \mu}\right)\right]}{\tau^{2}-\bar{\Omega}_{m \mu}^{2}} \mathrm{~d} \tau\right]=\frac{2 \sqrt{ } 2 \bar{\Omega}_{m \mu}}{U_{m \mu}(1)}
$$

since $L\left(\alpha_{m \mu}\right)=0$ and $L^{\prime}\left(\alpha_{m \mu}\right)=\frac{4}{\alpha_{m \mu} U_{m \mu}^{2}(1)}$; then if $k \rightarrow \alpha_{01} \beta=0$,

$$
\tilde{K}_{-}\left(\bar{\Omega}_{01}\right)=\tilde{K}_{-}(1) \cong \exp \left[\frac{\mathrm{i}}{\pi} \oint_{0}^{\infty} \frac{\log \left(1-h^{2}\right)}{\tau^{2}-1} d \tau\right]=\sqrt{1-h^{2}}
$$

furthermore, it is easily verified that the limits $\lim _{k \rightarrow \alpha_{m \mu} \beta \neq 0} \tilde{K}_{-}\left(\bar{\Omega}_{m \nu}\right)$ and $\lim _{k \rightarrow 0} \tilde{K}_{-}\left(\bar{\Omega}_{0 \mu}\right)$ exist. Applying these results in the foregoing expression for $R_{m \mu \nu}$ then yields, if $k \rightarrow \alpha_{m \mu} \beta$, $R_{m \mu \mu}=-1$ and $R_{m \mu \nu}=0$, if $k \rightarrow \alpha_{m \nu} \beta$,

$$
R_{m \mu \nu}=-\frac{1}{2} \sqrt{ } 2 U_{m \mu}(1) \frac{1+\bar{\Omega}_{m \mu}}{\bar{\Omega}_{m \mu}} \frac{1-M \gamma \bar{\Omega}_{m \mu}}{1-M \bar{\Omega}_{m \mu}} \tilde{K}_{-}\left(\bar{\Omega}_{m \mu}\right),
$$

and if $k \rightarrow \alpha_{m \eta} \beta, R_{m \mu \nu}=\cdots$ (no special form resulting). These forms are valid except when $m=0$ and $k \rightarrow \alpha_{01} \beta=0$, in which case

$$
\begin{gathered}
R_{011}=-\frac{1+M-2 M \gamma}{1-M}, \quad R_{01 \nu}=\frac{1}{2} \sqrt{ } 2 U_{0 \nu}(1) \frac{M}{1-M}(\gamma-1) \lim \left[\tilde{K}_{-}\left(\bar{\Omega}_{0 \nu}\right)\right], \\
R_{0 \mu 1}=\left\{\begin{array}{ll}
-\frac{1}{2} \sqrt{ } 2 \gamma U_{0 \mu}(1) \lim \left[\tilde{K}_{-}\left(\bar{\Omega}_{0 \mu}\right)\right] & \text { if } M \neq 0 \\
-\frac{1}{2} \sqrt{ } 2 U_{0 \mu}(1) \lim \left[\tilde{K}_{-}\left(\bar{\Omega}_{0 \mu}\right)\right] & \text { if } M=0
\end{array}\right\}, \\
R_{0 \mu \nu}=\left\{\begin{array}{ll}
-\frac{1}{4} U_{0 \mu}(1) U_{0 \nu}(1) \frac{\gamma+(\gamma-1) \alpha_{0 \nu} / \alpha_{0 \mu}}{1+\alpha_{0 \nu} / \alpha_{0 \mu}} \lim \left[\tilde{K}_{-}\left(\bar{\Omega}_{0 \mu}\right) \tilde{K}_{-}\left(\bar{\Omega}_{0 \nu}\right)\right] & \text { if } M \neq 0 \\
-\frac{1}{4} U_{0 \mu}(1) U_{0 \nu}(1) \frac{1}{1+\alpha_{0 \nu} / \alpha_{0 \mu}} \lim \left[\tilde{K}_{-}\left(\bar{\Omega}_{0 \mu}\right) \tilde{K}_{-}\left(\bar{\Omega}_{0 \nu}\right)\right] & \text { if } M=0
\end{array}\right\} .
\end{gathered}
$$

An expression analogous to the one for $R_{0 \mu \nu}$ and $k \rightarrow 0$ can be obtained for $R_{m \mu \nu}$ with $m \neq 0$.

\section{THE FAR FIELD}

When the scattering sound field sheds vorticity, these vortices are convected downstream together with an irrotational inherent velocity field. This field is non-decaying in the downstream direction, and therefore contributes to the far field. However, it is purely hydrodynamic (involves no pressure variations), is only significant near the wake and will therefore be treated separately from the acoustic far field. Hence one can start with a description of the acoustic far field. 
From the inverse transform of $\psi$ the pressure field for $r>1$ is

$$
p(x, r)=\frac{k^{2}}{2 \pi \mathrm{i}} \int_{-\infty-\mathrm{i} 0}^{\infty+\mathrm{i} 0}(1-u M) \Psi(u, r) \mathrm{e}^{-\mathrm{i} k u x} \mathrm{~d} u, \quad \Psi(u, r)=F_{+}(u) \frac{\mathrm{H}_{m}^{(2)}(k w r)}{k w \mathrm{H}_{m}^{(2) \prime}(k w)} .
$$

Introducing the transformations $\tau=\beta^{2} u+M, \quad v(\tau)=\beta w(u), \quad x=\beta R \cos \xi$ and $r=R \sin \xi, 0<\xi<\pi$, gives

$$
\begin{aligned}
p(x, r)= & \frac{1}{2 \pi \mathrm{i}} \frac{k}{\beta^{3}} \exp \left(\mathrm{i} M \frac{k}{\beta} R \cos \xi\right) \int_{-\infty-\mathrm{i} 0}^{\infty+\mathrm{i} 0}(1-M \tau) F_{+}\left(\frac{\tau-M}{\beta^{2}}\right) \\
& \times \frac{\mathrm{H}_{m}^{(2)}(v(\tau)(k R / \beta) \sin \xi)}{v(\tau) \mathrm{H}_{m}^{(2) \prime}(k v(\tau) / \beta)} \exp \left(-\mathrm{i} \tau \frac{k}{\beta} R \cos \xi\right) \mathrm{d} \tau .
\end{aligned}
$$

The acoustic far field, defined as the leading order term of the asymptotic expansion of $p$ for $(k R / \beta) \rightarrow \infty$ and $\xi$ fixed, can now be derived by means of the method of stationary phase [10, p. 34]. It is assumed explicitly that $\xi \neq 0$ and $\xi \neq \pi$. Then, for $v(\tau) \neq 0$,

$$
\mathrm{H}_{m}^{(2)}\left(v \frac{k}{\beta} R \sin \xi\right) \cong \mathrm{i}^{m}\left(\frac{2 \beta}{\pi v k R \sin \xi}\right)^{1 / 2} \exp \left(-\mathrm{i} v \frac{k}{\beta} R \sin \xi+\frac{1}{4} \pi \mathrm{i}\right) \quad\left(\frac{k}{\beta} R \rightarrow \infty\right) .
$$

The condition $v(\tau) \neq 0$ is easily achieved by indenting the integration contour a little into $R_{+}$near $\tau=1$, and into $R_{-}$near $\tau=-1$. Now consider the exponential part of the integrand, if $k R / \beta \rightarrow \infty$. This is $\exp [-\mathrm{i}(k / \beta) R(\tau \cos \xi+v(\tau) \sin \xi)]$. Clearly, the point of stationary phase is $\tau=\cos \xi$. With $k R / \beta$ large, the main contribution to the integral is then from the neighbourhood of this point, since away from this point the argument of the exponent is either purely imaginary, giving a rapidly oscillating integrand (if $-1<\tau<1$, real), or has a large negative real part, giving an exponentially small integrand (if $|\tau|>1$, real, or on the indentations). The appropriate calculations then give

$$
\begin{aligned}
& p(x, r) \cong \frac{\mathrm{i}^{m}}{\pi \beta^{2}} \frac{(1-M \cos \xi) F_{+}\left[(\cos \xi-M) / \beta^{2}\right] \exp [-\mathrm{i}(k R / \beta)(1-M \cos \xi)]}{R \sin \xi \mathrm{H}_{m}^{(2) \prime}[(k / \beta) \sin \xi]} \\
& \quad\left(\frac{k}{\beta} R \rightarrow \infty\right) .
\end{aligned}
$$

A common way to characterize this far field is by a directivity function $D_{m \mu}(\xi)$, defined by

$$
\begin{gathered}
p(x, r) \cong D_{m \mu}(\xi) \exp [-\mathrm{i}(k R / \beta)(1-M \cos \xi)] /(k R / \beta), \\
D_{m \mu}(\xi)=\frac{\mathrm{i}^{m} k}{2 \pi \beta} U_{m \mu}(1) \tilde{K}_{-}\left(\bar{\Omega}_{m \mu}\right) \frac{1+\bar{\Omega}_{m \mu}}{1-M \bar{\Omega}_{m \mu}} \frac{(1-\cos \xi) \tilde{K}_{+}(\cos \xi)}{\sin \xi \mathrm{H}_{m}^{(2) \prime}[(k / \beta) \sin \xi]}\left[\frac{1-M \cos \xi}{\bar{\Omega}_{m \mu}-\cos \xi}-M \gamma\right] .
\end{gathered}
$$

Another way to introduce a directivity function is, in physical co-ordinates $x=\bar{R} \cos \bar{\xi}$ and $r=\bar{R} \sin \bar{\xi}$, as

$$
\begin{gathered}
p(x, r) \cong E_{m \mu}(\bar{\xi}) \exp \left[-\mathrm{i}\left(k \bar{R} / \beta^{2}\right)\left(\sqrt{1-M^{2} \sin ^{2} \bar{\xi}}-M \cos \bar{\xi}\right)\right] /(k \bar{R} / \beta), \\
E_{m \mu}(\bar{\xi})=\frac{\beta}{\left(1-M^{2} \sin ^{2} \bar{\xi}\right)^{1 / 2}} D_{m \mu}\left(\arccos \frac{\cos \bar{\xi}}{\left(1-M^{2} \sin ^{2} \bar{\xi}\right)^{1 / 2}}\right) .
\end{gathered}
$$

On the other hand, in some applications the so called emission time co-ordinates are more relevant, $x=R_{e}\left(M+\cos \xi_{e}\right)$ and $r=R_{e} \sin \xi_{e}$, giving

$$
p(x, r) \cong D_{m \mu}^{e}\left(\xi_{e}\right) \frac{\exp \left[-\mathrm{i} k R_{e}\right]}{k R_{e}}, \quad D_{m \mu}^{e}\left(\xi_{e}\right)=\frac{\beta^{2}}{1+M \cos \xi_{e}} D_{m \mu}\left(\arccos \frac{M+\cos \xi_{e}}{1+M \cos \xi_{e}}\right)
$$


The following properties of $D_{m \mu}$ are worth mentioning. For $0<\xi<\pi / 2$ the radiation pattern, i.e., $\left|D_{m \mu}\right|$, consists of lobes, whereas for $\pi / 2 \leqslant \xi<\pi$ it is free of zeros, and tends to zero for $\xi \rightarrow \pi$ if $m \geqslant 1$, and to a finite value if $m=0$. These lobes are formed by maxima, interlaced by zeros: $D_{m \mu}(\xi)=0$ at $\xi=\arccos \bar{\Omega}_{m \nu}, \nu \neq \mu, \bar{\Omega}_{m \nu}$ real. If the incident wave is cut-on, i.e., $\bar{\Omega}_{m \mu}$ is real, then

$$
D_{m \mu}(\xi)=\frac{2 \mathrm{i}^{m} k^{2} \bar{\Omega}_{m \mu}}{\pi \beta^{2} \alpha_{m \mu} U_{m \mu}(1) \mathrm{H}_{m}^{(2) \prime}\left(\alpha_{m \mu}\right)} \quad \text { at } \xi=\arccos \bar{\Omega}_{m \mu}
$$

In particular $\lim _{\xi \downarrow 0} D_{01}(\xi)=\frac{1}{2} \sqrt{ } 2 \mathrm{i}(k / \beta)^{2} \sqrt{1-h^{2}}$; otherwise, $\lim _{\xi \downarrow 0} D_{m \mu}(\xi)=0$.

Furthermore, one can quantify the effect of vortex shedding on the directivity pattern by considering $\left.D_{m \mu}(\xi)\right|_{\gamma=1} /\left.D_{m \mu}(\xi)\right|_{\gamma=0}=\left(1-M \bar{\Omega}_{m \mu}\right) /(1-M \cos \xi)$. It is evident that there is an increase, for $0<\xi<\arccos \bar{\Omega}_{m \mu}$ if $\alpha_{m \mu}<k \beta^{-1}$, for $0<\xi<$ $\arccos \left(\left[1-\left(1+M^{2}\left|\bar{\Omega}_{m \mu}\right|^{2}\right)^{1 / 2}\right] / M\right)$ if $k \beta^{-1}<\alpha_{m \mu} \leqslant \sqrt{ } 2 k[M(1-M)]^{-1 / 2}$, and for all $\xi$ if $\alpha_{m \mu}>\sqrt{ } 2 k[M(1-M)]^{-1 / 2}$, and there is a decrease in each complementary angle interval. This is analogous to the behaviour found in the two-dimensional variant of the present problem [11].

By using the results derived in the previous section it is easily seen that near cut-off the behaviour of $D_{m \mu}$ is that $D_{m \mu}(\xi)$ tends to zero if $k \rightarrow \alpha_{m \mu} \beta$, or if $k \rightarrow 0$, and $D_{m \mu}(\xi)$ tends to a finite limit if $k \rightarrow \alpha_{m \nu} \beta \neq 0(\mu \neq \nu)$.

Next one can consider the hydrodynamic far field. Since no pressure is associated with it, one has to return to the potential $\phi$, in its form of an inverse Fourier transform of $\psi$. As $x$ tends to infinity, the main contribution to the integral is now from the pole in $u=M^{-1}$, so that if $h \leqslant r \leqslant 1, x \rightarrow \infty$,

$$
\phi(x, r) \cong \gamma C_{m \mu}^{H} \frac{\mathrm{K}_{m}^{\prime}(k h / M) \mathrm{I}_{m}(k r / M)-\mathrm{I}_{m}^{\prime}(k h / M) \mathrm{K}_{m}(k r / M)}{\mathrm{K}_{m}^{\prime}(k h / M) \mathrm{I}_{m}^{\prime}(k / M)-\mathrm{I}_{m}^{\prime}(k h / M) \mathrm{K}_{m}^{\prime}(k / M)} \exp \left(-\mathrm{i} \frac{k}{M} x\right),
$$

and if $1 \leqslant r<\infty, x \rightarrow \infty$,

$$
\phi(x, r) \cong \gamma C_{m \mu}^{H}\left[\mathrm{~K}_{m}(k r / M) / \mathrm{K}_{m}^{\prime}(k / M)\right] \exp (-\mathrm{i} k x / M),
$$

where the constant

$$
C_{m \mu}^{H}=\frac{1}{2} \mathrm{i}(1-M) U_{m \mu}(1) \tilde{K}_{-}\left(\bar{\Omega}_{m \mu}\right) \tilde{K}_{+}\left(M^{-1}+\mathrm{i} 0\right)\left(1+\bar{\Omega}_{m \mu}\right) /\left(1-M \bar{\Omega}_{m \mu}\right),
$$

and $\mathrm{K}_{m}$ and $\mathrm{I}_{m}$ denote modified Bessel functions of $m$ th order.

It is seen that this field does not decay in $x$, and behaves exponentially in $r$ in a way that confines it to the immediate neighbourhood of the wake. Note that the characteristic length scale in both the $x$ and $r$ directions is the inverse of the Strouhal number (or hydrodynamic wave number) $k / M$.

\section{ACOUSTIC AND HYDRODYNAMIC POWER}

A quantity of interest is the power of the field inside and outside the pipe, since it characterizes, to a certain extent, the field by a single number. The power is defined as the net (integrated), time-averaged, energy flux across a control surface. Useful surfaces here are a large sphere around the pipe exit, a cross section of the pipe, and in the case of vortex shedding, two cylindrical surfaces enclosing the wake, since there the power is both physically interesting and explicitly computable. In general, in a moving medium an acoustic energy obeying a conservation equation cannot be defined by using only linear quantities, since quantities of second order may couple to zeroth order quantities (mean flow terms) giving contributions of acoustic order unknown from the linear acoustics 
alone. Only under certain restrictions can a conserved energy be defined; an example relevant here is an irrotational field in an irrotational main flow. The expression in question can be found, for instance, in reference [9, p. 41], and results, in the present terminology, in a power $P=\int_{S}(\vec{I} \cdot \vec{n}) \mathrm{d} s$, made dimensionless on $\rho_{0}^{*} c_{0}^{*^{3}} b^{* 2}$, where $S$ is the control surface considered with normal vector $\vec{n}$, and $\vec{I}$ is the (dimensionless) acoustic intensity (三time-averaged energy flux) $\vec{I}=\frac{1}{2} k \operatorname{Im}\left\{\phi \vec{\nabla} \phi^{c}+M \phi p^{c} \vec{e}_{x}\right\}$, with superscript $c$ indicating the complex conjugate, and $\vec{e}_{x}$ the unit vector in the $x$-direction. Also, indeed, for the present problem (with only an acoustic vortex sheet) no general definition of energy based on the acoustic variables alone is possible, and the restriction of this expression to irrotational regions is essential, as was shown by Rienstra [11] for an explicit (2-D) example. In this case, the acoustic field appeared to be coupled to the mean flow, through the vortex shedding, in such a way that not only energy is exchanged between the acoustic field and the vorticity, but also with the mean flow. Therefore, the control surfaces in the present problem must not cross the vortex sheet anywhere, and also one cannot expect a global conservation of energy. It is, however, possible to follow the energy transfer through the various parts of the field, and to analyze the energy conversion associated to the vortex shedding in detail. To this end one can investigate (i) in the absence of vortex shedding $(\gamma=0)$, the transmitted power $P^{t}$ inside the pipe, and the power $P^{f}$ of the far field; since the field is now irrotational everywhere, energy is conserved, so $P^{t}=P^{f}$; (ii) in the presence of vortex shedding $(\gamma \neq 0)$, again the transmitted power $P^{t}$ and the power $P^{f}$ of the acoustic far field (i.e., not near the wake), but also the power of the hydrodynamic far field downstream, inside $\left(P_{i}^{H}\right)$ and outside $\left(P_{o}^{H}\right)$ the cylinder formed by the vortex sheet, and the power $P^{w}$ lost into the wake, i.e., the difference of the power just inside and just outside the vortex sheet; since the only possible extra source or sink of acoustic energy (directly, or through interaction with the main stream) is the wake, the power balance can be formulated as $P^{t}=P^{f}+P^{w}+P_{i}^{H}+P_{o}^{H}$, which yields a very useful check, and has been applied to verify the numerical calculations. Although there is an essential difference between the loss of acoustic energy into the irrotational hydrodynamic far field $\left(P^{H}\right)$ and into the wake $\left(P^{w}\right)$, clearly in many applications only the net acoustic result is of interest. $\dagger$

In view of the occurrence of quadratic terms in a secondary quantity, such as power, it is appropriate to consider a general time harmonic incident wave (each mode of which has amplitude $A_{m \mu}$ ), yielding inside the pipe the field

$$
\begin{aligned}
p(x, r, \theta) & =\sum_{m=-\infty}^{\infty} \sum_{\mu=1}^{\infty}\left\{A_{m \mu} \exp \left(-\mathrm{i} k_{m \mu}^{+} x\right)+B_{m \mu} \exp \left(-\mathrm{i} k_{m \mu}^{-} x\right)\right\} U_{m \mu}(r) \mathrm{e}^{\mathrm{i} m \theta}, \\
B_{m \mu} & =\sum_{\nu=1}^{\infty} A_{m \nu} R_{m \nu \mu}, \quad U_{-m \mu}(r)=(-1)^{m} U_{m \mu}(r), \quad k_{-m \mu}^{ \pm}=k_{m \mu}^{ \pm} .
\end{aligned}
$$

By taking advantage of the orthogonality properties of $\left\{\mathrm{e}^{\mathrm{i} m \theta}\right\}$ on $[0,2 \pi)$ and $\left\{U_{m \mu}(r)\right\}$ on $[h, 1]$, it is readily found that

$$
\begin{aligned}
P^{t}= & \pi \beta^{4} \sum_{m=-\infty}^{\infty} \sum_{\mu=1}^{\mu_{0}-1} \bar{\Omega}_{m \mu}\left[\frac{\left|A_{m \mu}\right|^{2}}{\left(1-M \bar{\Omega}_{m \mu}\right)^{2}}-\frac{\left|B_{m \mu}\right|^{2}}{\left(1+M \bar{\Omega}_{m \mu}\right)^{2}}\right] \\
& +2 \pi \beta^{4} \sum_{m=-\infty}^{\infty} \sum_{\mu=\mu_{0}}^{\infty} \frac{\left|\bar{\Omega}_{m \mu}\right|}{\left(1+M^{2}\left|\bar{\Omega}_{m \mu}\right|^{2}\right)^{2}}\left[\operatorname{Im}\left(A_{m \mu} B_{m \mu}^{c}\right)\left(1-M^{2}\left|\bar{\Omega}_{m \mu}\right|^{2}\right)\right. \\
& \left.-\operatorname{Re}\left(A_{m \mu} B_{m \mu}^{c}\right) 2 M\left|\bar{\Omega}_{m \mu}\right|\right]
\end{aligned}
$$

$\dagger$ It is noted that in reference [11] only the $P^{w}$ was considered, not $P^{H}$. $P^{w}$ represents the energy that really disappears "out of the world". 
where a superscript $c$ denotes a complex conjugate, and $\mu_{0}=\mu_{0}(m)$ indicates the index of the first cut-off mode (the first with $\bar{\Omega}_{m \mu}$ negative imaginary; see also reference [12]).

In the acoustic far field the sound waves radiate, apart from the convection effects, radially outwards. Therefore, one can obtain in the far field the potential and velocity from the pressure as follows:

$$
\begin{aligned}
& \phi \cong \frac{\mathrm{i} \beta^{2}}{k(1-M \cos \xi)} p, \quad \phi_{x} \cong \frac{\cos \xi-M}{1-M \cos \xi} p, \quad \phi_{r} \cong \frac{\beta \sin \xi}{1-M \cos \xi} p, \\
& p(x, r, \theta) \cong \sum_{m=-\infty}^{\infty} \sum_{\mu=1}^{\infty} A_{m \mu} D_{m \mu}(\xi) \frac{\exp [\mathrm{i} m \theta-\mathrm{i}(k R / \beta)(1-M \cos \xi)]}{k R / \beta} .
\end{aligned}
$$

A convenient control surface for $P^{f}$ is a large ellipsoid $R=$ constant. On this surface

$$
(\vec{I} \cdot \vec{n})=\frac{\frac{1}{2} \beta^{4}}{(1-M \cos \xi)^{2}\left(1-M^{2} \sin ^{2} \xi\right)^{1 / 2}}|p|^{2} .
$$

Now consider the parametrization of the ellipsoid $\vec{E}(\xi, \theta)=\beta R \cos \xi \vec{e}_{x}+R \sin \xi \cos \theta$ $\vec{e}_{y}+R \sin \xi \sin \theta \vec{e}_{z}$, where $\vec{e}_{y}$ and $\vec{e}_{z}$ are unit vectors in the $y$ and $z$ directions $(y=r \cos \theta$, $z=r \sin \theta)$. A well-known result from calculus is that the surface integral of $(\vec{I} \cdot \vec{n})$ over the ellipsoid is given by

$$
\int_{E}(\vec{I} \cdot \vec{n}) \mathrm{d} s=\int_{0}^{2 \pi} \int_{0}^{\pi}(\vec{I} \cdot \vec{n})\left|\vec{E}_{\xi} \times \vec{E}_{\theta}\right| \mathrm{d} \xi \mathrm{d} \theta .
$$

Hence, since $\left|\vec{E}_{\xi} \times \vec{E}_{\theta}\right|=R^{2} \sin \xi\left(1-M^{2} \sin ^{2} \xi\right)^{1 / 2}$, one has (utilizing the orthogonality of $\left.\left\{\mathrm{e}^{\mathrm{i} m \theta}\right\}\right)$

$$
\begin{aligned}
P^{f} & =\frac{1}{2} \beta^{4} R^{2} \int_{0}^{2 \pi} \int_{0}^{\pi} \frac{\sin \xi}{(1-M \cos \xi)^{2}}|p|^{2} \mathrm{~d} \xi \mathrm{d} \theta \\
& =\frac{\pi \beta^{6}}{k^{2}} \sum_{m=-\infty}^{\infty} \int_{0}^{\pi} \frac{\sin \xi}{(1-M \cos \xi)^{2}}\left|\sum_{\mu=1}^{\infty} A_{m \mu} D_{m \mu}(\xi)\right|^{2} \mathrm{~d} \xi .
\end{aligned}
$$

It is evident that for both $P^{t}$ and $P^{f} m$-mode contributions simply can be summed; no cross interference occurs. This is, however, not the case for $\mu$-mode contributions. In the expression for $P^{t}$ a coefficient $B_{m \mu}$ is formed by all the occurring $A_{m \mu}$ 's, giving non-zero cross terms in $\left|B_{m \mu}\right|^{2}$. In view of conservation of energy (i.e., $P^{t}=P^{f}$ ) if $\gamma=0$, the cross terms from the squared $\mu$-series of the integrand of $P^{f}$, when integrated, will then definitely produce non-zero values too.

On the other hand, no cross interference occurs between the acoustic and the hydrodynamic far field, this hydrodynamic field being only significant within a $\xi$-interval of vanishing size. So the hydrodynamic powers $P_{i}^{H}$ and $P_{o}^{H}$ can be calculated immediately as

$$
\begin{aligned}
P_{i}^{H}= & \int_{0}^{2 \pi} \int_{h}^{1} \frac{k^{2}}{2 M}|\phi(x, r, \theta)|^{2} r \mathrm{~d} r \mathrm{~d} \theta=\frac{1}{2} \pi M|\gamma|^{2} \sum_{m=-\infty}^{\infty}\left|\sum_{\mu=1}^{\infty} A_{m \mu} C_{m \mu}^{H}\right|^{2} \\
& \times\left\{\left(m^{2}+\frac{k^{2}}{M^{2}}\right)\left[\frac{\mathrm{K}_{m}^{\prime}(k h / M) \mathrm{I}_{m}(k / M)-\mathrm{I}_{m}^{\prime}(k h / M) \mathrm{K}_{m}(k / M)}{\mathrm{K}_{m}^{\prime}(k h / M) \mathrm{I}_{m}^{\prime}(k / M)-\mathrm{I}_{m}^{\prime}(k h / M) \mathrm{K}_{m}^{\prime}(k / M)}\right]^{2}-\frac{k^{2}}{M^{2}}\right. \\
& \left.-\left(m^{2}+\frac{k^{2} h^{2}}{M^{2}}\right)\left[\frac{M / k h}{\mathrm{~K}_{m}^{\prime}(k h / M) \mathrm{I}_{m}^{\prime}(k / M)-\mathrm{I}_{m}^{\prime}(k h / M) \mathrm{K}_{m}^{\prime}(k / M)}\right]^{2}\right\}
\end{aligned}
$$




$$
\begin{aligned}
P_{0}^{H} & =\int_{0}^{2 \pi} \int_{1}^{\infty} \frac{k^{2}}{2 M}|\phi(x, r, \theta)|^{2} r \mathrm{~d} r \mathrm{~d} \theta \\
& =\frac{1}{2} \pi M|\gamma|^{2} \sum_{m=-\infty}^{\infty}\left|\sum_{\mu=1}^{\infty} A_{m \mu} C_{m \mu}^{H}\right|^{2}\left\{\frac{k^{2}}{M^{2}}-\left(m^{2}+\frac{k^{2}}{M^{2}}\right) \frac{\mathrm{K}_{m}(k / M)^{2}}{\mathrm{~K}_{m}^{\prime}(k / M)^{2}}\right\} .
\end{aligned}
$$

Finally, the net power absorbed by the wake,

$$
P^{w}=-\frac{1}{2} k \int_{0}^{\infty} \int_{0}^{2 \pi} \operatorname{Im}\left[\left\{\phi^{c}(x, 1-, \theta)-\phi^{c}(x, 1+, \theta)\right\} \frac{\partial}{\partial r} \phi(x, 1, \theta)\right] \mathrm{d} \theta \mathrm{d} x,
$$

can be calculated explicitly if one observes that the difference of the potential across the wake is dependent on $x$ only through a factor $\exp \left(-\mathrm{i} k M^{-1} x\right)$. This implies that, after performing the relatively simple $\theta$-integral, the remaining $x$-integral is very similar to the Fourier transform of $(\partial / \partial r) \phi(x, 1, \theta)$, viz. $F_{+}(u)$, in $u=M^{-1}$. The difference is the imaginary part to be taken here. Unfortunately, $F_{+}(u)$ is singular in $u=M^{-1}$. Some more detailed analysis, however, reveals that this singularity contributes to the real part only, and one obtains

$$
\begin{aligned}
P^{w}= & \frac{1}{2} \pi \beta^{2}(1-M)^{2} \operatorname{Re} \sum_{m=-\infty}^{\infty} \sum_{\mu=1}^{\infty} \sum_{\nu=1}^{\infty}\left[A_{m \mu} U_{m \mu}(1) \frac{1+\bar{\Omega}_{m \mu}}{1-M \bar{\Omega}_{m \mu}} \frac{\tilde{K}_{-}\left(\bar{\Omega}_{m \mu}\right)}{\tilde{K}_{-}\left(M^{-1}\right)}\right] \\
& \times\left[A_{m \nu} U_{m \nu}(1) \frac{1+\bar{\Omega}_{m \nu}}{1-M \bar{\Omega}_{m \nu}} \frac{\tilde{K}_{-}\left(\bar{\Omega}_{m \nu}\right)}{\tilde{K}_{-}\left(M^{-1}\right)}\right]^{c} \\
& \times\left[\left(\frac{\gamma^{c}}{1-M \bar{\Omega}_{m \mu}}-\frac{|\gamma|^{2}}{1-M}\right) M \tilde{K}\left(M^{-1}\right)-\mid \gamma_{1}^{2} \tilde{K}_{+}^{\prime}\left(M^{-1}\right) \tilde{K}_{-}\left(M^{-1}\right)\right] .
\end{aligned}
$$

The argument $M^{-1}$ is located above the branch cut but below the integration contour, symbolically denoted by $M^{-1}+\mathrm{i} 0$. Note that $\tilde{K}\left(M^{-1}+\mathrm{i} 0\right)=L(-\mathrm{i} k / M)$ is real. By definition $\tilde{K}_{+}^{\prime}\left(M^{-1}\right)=(\mathrm{d} / \mathrm{d} \tau) \tilde{K}_{+}(\tau)$ is in $\tau=M^{-1}+\mathrm{i} 0$. Effectively, one can say that these expressions for $P^{w}, P_{i}^{H}$ and $P_{o}^{H}$ together with $P^{t}$ provide an explicit form of the integral of $P^{f}\left(=P^{t}-P^{w}-P_{i}^{H}-P_{o}^{H}\right)$. Comparison of $P^{w}$ with $P_{i}^{H}$ and $P_{o}^{H}$ shows a dependence of all on $|\gamma|^{2}$, but of $P^{w}$ on $\gamma$ as well. This implies that with little vorticity shed $(|\gamma| \ll 1)$ the only exchange of acoustic energy consists of an absorption by the wake without conversion into the irrotational hydrodynamic field.

All in all, the conclusion that energy can be absorbed or emitted by the vortex sheet is remarkable. The transfer mechanism appears to depend on the mutual interference of the acoustic field at the vortex sheet and the convected unsteady vorticity.

In a following section numerically evaluated examples are discussed, including the acoustic power loss due to vortex shedding, but, for practical reasons, without distinguishing between the irrotational hydrodynamic power and the absorption by the wake. Some idea of the possible relative importance of the contributions can be provided by considering an illustrative example. For one incident mode, a plane wave with $m=0, \mu=1, A_{01}=1$, $k=1$ in a pipe with $h=0.5$ and a flow of $M=0.5$ and $\gamma=1$, it is found that $P^{t}=6 \cdot 6525$, $P^{f}=1 \cdot 3484, P^{w}=1 \cdot 8064, P_{i}^{H}=2 \cdot 7993$ and $P_{o}^{H}=0 \cdot 6984$, all of which contribute significantly.

\section{NUMERICAL EVALUATION OF $\tilde{K}_{-}(r)$}

To evaluate the results of the present analysis, a computer program was written. The basic problem was to calculate $\tilde{K}_{ \pm}$, from which straightforwardly the reflection matrix 
[ $\left.R_{m \mu \nu}\right]$, the directivity pattern $D_{m \mu}(\xi)$ and for a given incident wave, the powers $P^{t}$ and $P^{f}$ are obtained. Details of the computer program are not given. Some analytical aspects concerning the integral for $\tilde{K}_{ \pm}$are as follows.

First, it is only necessary to consider $\tilde{K}_{-}(t)$ with $-\pi<\arg t \leqslant 0 . \tilde{K}_{+}(t)$ follows by definition from $\tilde{K}_{+}(t)=\tilde{K}(t) / \tilde{K}_{-}(t)$ while if $0<\arg t \leqslant \pi$ one can use the symmetry of $\tilde{K}(t)$ to have $\tilde{K}_{+}(t)=\tilde{K}_{-}(-t)$ and $\tilde{K}_{-}(t)=\tilde{K}(t) / \tilde{K}_{-}(-t)$. Thus

$$
\log \tilde{K}_{-}(t)=-\frac{1}{2 \pi \mathrm{i}} \int_{-\infty-\mathrm{i} 0}^{\infty+\mathrm{i} 0} \frac{\log \tilde{K}(\tau)}{\tau-t} \mathrm{~d} \tau, \quad-\pi<\arg t \leqslant 0,
$$

the contour of integration crossing the real axis at $\tau=0$. Two difficulties are now immediately apparent. The first is that the integrand behaves at infinity like $\log \tau / \tau$, so the integral does not converge, while the second is that the integrand has singularities along the contour of integration (viz. along $[-1,1]$ ); so, upper (along $(0,1])$ and lower (along $[-1,0)$ ) indentations around these singularities are to be taken. The first problem is only minor, and can be dealt with as by Noble [10, p. 42], by defining the integral as the limit $\lim _{A \rightarrow \infty} \int_{-A}^{A} \cdots \mathrm{d} \tau$. After taking advantage of the symmetry of $\tilde{K}(\tau)$, one obtains

$$
\log \tilde{K}_{-}(t)=\frac{\mathrm{i} t}{\pi} \int_{0}^{\infty} \frac{\log \tilde{K}(\tau)}{\tau^{2}-t^{2}} \mathrm{~d} \tau, \quad-\pi<\arg t \leqslant 0 .
$$

The second problem is, as is usual in this kind of work, mostly solved by a delicate process of splitting off and handling separately each singularity, followed by a tedious and cumbersome bookkeeping of improper integrals $[4,6,10,13,14]$. However, it has been found to be much more convenient to deform the contour of integration between $\tau=0$ and $A>1$ into a parabola-like contour in the upper half plane $(\operatorname{Im} \tau=\operatorname{Re} \tau(1-\operatorname{Re} \tau / A)$, see Figure 3), in this way avoiding all the singularities along $0<\tau \leqslant 1$, without altering

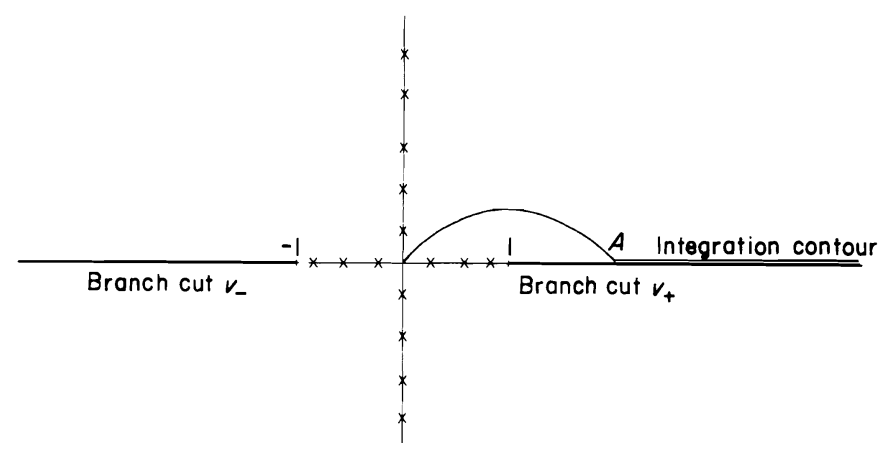

Figure 3. The complex $\tau$ plane, with deformed contour of integration, zeros $\pm \bar{\Omega}_{m \mu}(\times)$, and branch cuts of $v_{+}$and $v_{-}$.

the value of the integral, since the integrand is analytic in that area. This can be seen as follows: since the values of $t$ relevant here are either real or imaginary, the pole in $\tau=t$ is not crossed; the zeros of $\mathrm{J}_{m}^{\prime}(z h) \mathrm{Y}_{m}^{\prime}(z)-\mathrm{Y}_{m}^{\prime}(z h) \mathrm{J}_{m}^{\prime}(z), z=k v(\tau) / \beta$, are found at $\tau= \pm \bar{\Omega}_{m \nu}$, which are only real or imaginary, while the pole of $z=0$ is at the real numbers $\tau= \pm 1$; the branch cuts, introduced via the square root $v(\tau)$, run along the real axis from \pm 1 to infinity, not through the origin; since $-\frac{1}{2} \pi<\arg v(\tau)<0$ if $0<\arg \tau<\frac{1}{2} \pi$ one has $\mathrm{H}_{m}^{(2) \prime}(k v / \beta)=-(2 / \pi) \mathrm{i}^{m} \mathrm{~K}_{m}^{\prime}(\mathrm{i} k v / \beta)$ (where $\mathrm{K}_{m}$ is the modified Bessel function of the second kind) so that one can use a corollary of a theorem of Erdélyi and Kermak [15, p. 62], implying that $\mathrm{K}_{m}^{\prime}(z)$ has no zeros in $|\arg z| \leqslant \frac{1}{2} \pi$. 
Thus the integral has been calculated numerically along a path through the complex plane (essentially by using complex Bessel function routines) up to a real point $\tau=A$. To minimize the amount of numerical work further this point $A$ was chosen just large enough (depending on the problem parameters) to admit the analytic integration along $[A, \infty)$ of an asymptotic approximation of the integrand. This approximation and its integration is given in detail in the Appendix.

Finally, acknowledgment must be made of a referee's kind remark that the same idea of a contour deformation to avoid the singularities was recently proposed by Ramakrishnan [16] in this journal. However, instead of one indentation around all the singularities he used one indentation for each, which may still be laborious at higher frequencies, with many poles along $[0,1]$. Moreover, he did not include a proof of the integrand being analytic in the region crossed.

\section{COMPUTED RESULTS}

To illustrate the present analysis with some numerically calculated examples, two ranges of frequency are considered: one with low values of $k(0<k<3)$, and one with a high value $(k=50)$. The Mach number was varied from $0 \cdot 2$ to $0 \cdot 8$, and the hub radius from 0.0 to 0.95 . The higher value of $k$ is typical of a modern turbofan engine. The lower value range is chosen to investigate the effect of sound absorption by vortex shedding, which is known to be strongest for the lower Helmholtz numbers $[17,18]$.

Figures 4(a), 5(a) and 6(a) show the power loss (in dB) $10_{10} \log \left(P^{f} / P^{t}\right)$ (due to the vortex shedding) of a plane incident wave $(m=0, \mu=1)$, for $M=0.2,0.5$ and 0.8 , respectively, as a function of $k$ (between 0 and 1) and $h$. Included in the figures is a result by Cargill [19] for the power loss in a jet with ambient flow, without a hub, with full Kutta condition, in a small Helmholtz number $(k)$ approximation. His general formula was

$$
P^{f} / P^{t}=\left(k^{2} / 4 M\right)\left(1+\frac{1}{3} M_{R}^{2}\right) /\left(1-M_{R}^{2}\right)^{3},
$$

where $M_{R}$ denotes the relative Mach number between jet and ambient flow $\left(0 \leqslant M_{R} \leqslant M\right)$. Shown in the figures are two cases: a uniform mean flow $\left(M_{R}=0\right)$, and a jet in a stagnant medium $\left(M_{R}=M\right)$. The formula is an approximation of Munt's full analytical solution [7] and for the jet $\left(M_{R}=M\right)$ known to be in very good agreement (in the lower $k$-range) both with Munt's solution and with the experiments of Bechert, Michel and Pfizenmaier [17]. From the figures the same good agreement is seen in the case of uniform flow (and $h=0)$.

A main observation by Bechert et al. [17] was a dramatic attenuation when large wavelength sound was radiated out of a jet pipe. From the figures the same effect is seen in a uniform flow, so this attenuation is not an exclusive property of the jet and has to do only with the unsteady vortex shedding. Even more, in a uniform flow the effect is much stronger, so it is not amplified but just weakened by the presence of the jet! Although indeed consistent with Cargill's work, this can be considered quite surprising.

Other trends to be noted are the increasing loss with increasing Mach number (not true in a jet if $M>0 \cdot 36$ ), and the increasing loss with increasing hub radius. This can be interpreted as another way to decrease the effective Helmholtz number, since the effective surface of a cross section of the tube can be decreased as well by taking $k \rightarrow 0$ as by $h \rightarrow 1$; in other words, the power loss scales upon $k^{2}\left(1-h^{2}\right)$ for small $k$.

In Figures 4(b), 5(b) and 6(b) the modulus of the reflection coefficient of the wave into its own mode $\left(\left|R_{011}\right|\right)$ is plotted. As was seen in section 4 this quantity tends to unity for $k \rightarrow 0$ in a way very similar to that without flow (cf. [14]). Apart from the "effective 

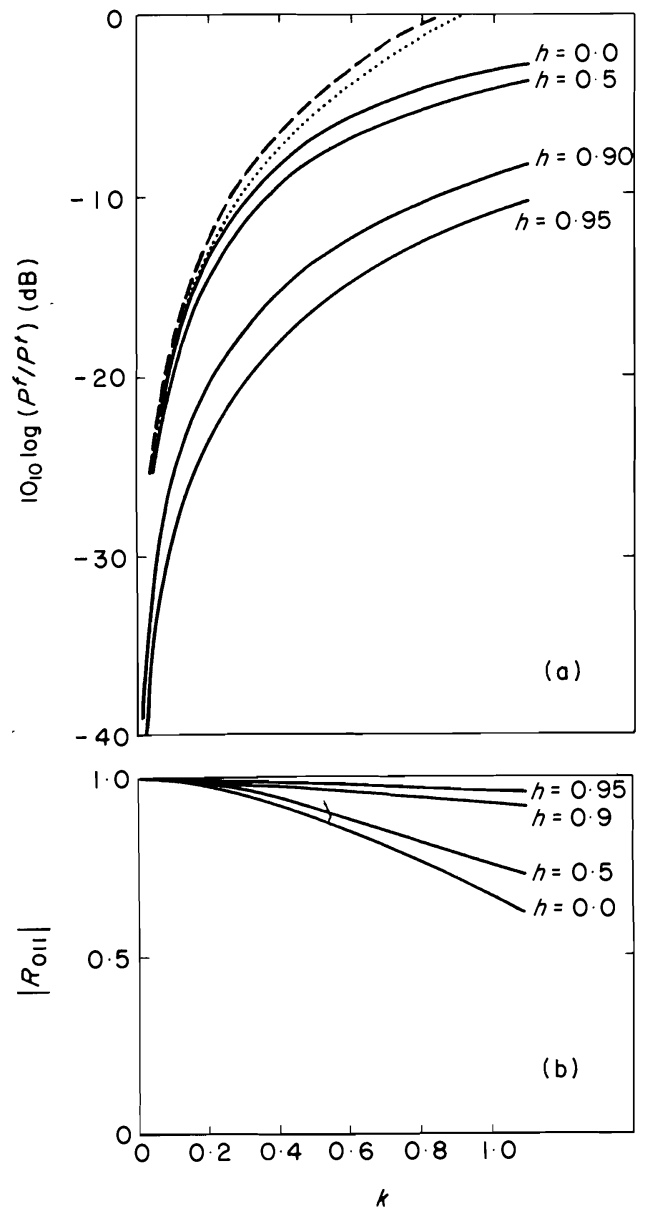

Figure 4. (a) Power loss $10_{10} \log \left(P^{f} / P^{t}\right)$ (in dB) of a plane incident wave $(m=0, \mu=1)$ for $M=0 \cdot 2$;

- , present results for uniform flow; $-\ldots, \cdots$, ,

Cargill's approximation for a jet and for uniform flow, respectively $(h=0)$; (b) modulus of reflection coefficient $\left|R_{011}\right|$ for same conditions as (a).
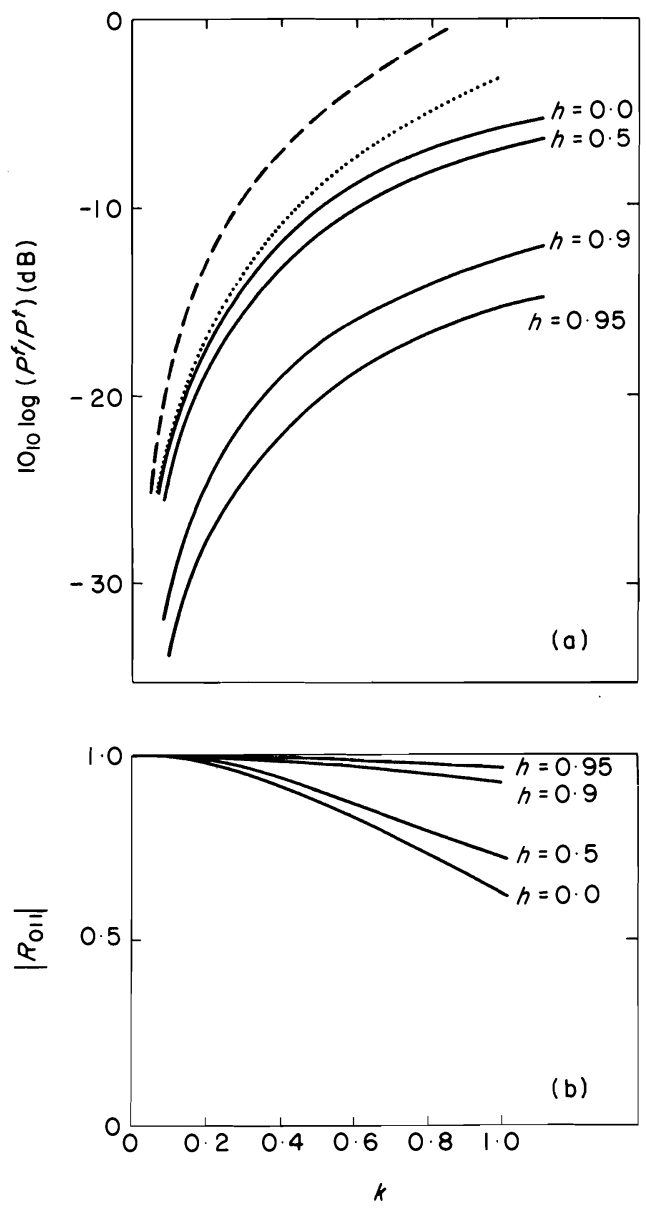

Figure 5. The same as Figures $4(a)$ and (b) but with $M=0 \cdot 5$.

Helmholtz number decreasing" effect of an increasing $h$, no special phenomena are observed.

A sequence analogous to the previous one is given in Figures 7,8 and 9, which are concerned with whether the large loss of power of the plane wave (Figures 4-6) near $k=0$ is primarily due to the frequency being near cut-off, or just to its being small. Figures 7(a), 8(a) and 9(a) show the power loss of an incident $m=1, \mu=1$ wave for values of $k$ between cut-off and 3. Here it is evident that the value of $k$ being near cut-off is not crucial. There is indeed a considerable power loss, but no asymptotic behaviour to minus infinity appears near cut-off; instead, the loss tends to a finite limit. Again an increasing mach number or hub diameter increases the loss (except for a small anomaly for $h=0.0$ and 0.5 , connected, for $\mu=1$, to the decreasing cut-off frequency with increasing $h$ ).

Figures 7(b), 8(b) and 9(b) show a reflection coefficient $\left|R_{111}\right|$ behaviour very similar to a case without flow (obviously, the cut-off frequencies are shifted with the Mach number). 

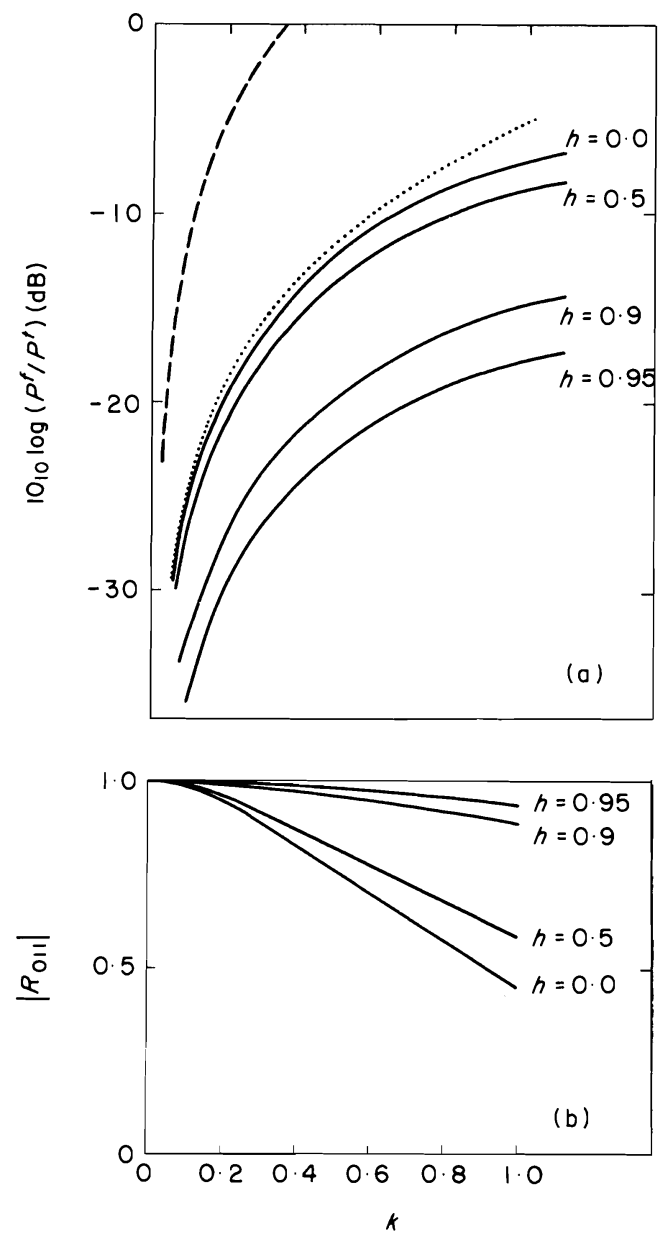

Figure 6. The same as Figures $4(\mathrm{a})$ and (b) but with $M=0 \cdot 8$.

In Figures 10,11 and 12 the far field radiation patterns $\left|E_{m \mu}\right|$ are plotted on a logarithmic scale for various inlet and outlet configurations $(k=50, m=4$ and $M= \pm 0 \cdot 3)$. In Figure 10 (a) a $\mu=1$, and in Figure 10 (b) a $\mu=2$, wave is radiating out of an inlet $(M=-0 \cdot 3$, hub diameter $=0$, no vortex shedding). As discussed in section 5 , the 15 possible cut-on modes are mirrored in the 14 zeros (the result of interference between waves scattered at the two sides of the duct), and a maximum lobe from the actual incident wave (here $\mu=1$ and $\mu=2$ ). Since the incident wave is not a plane wave, the pressure level tends to zero as $\xi \rightarrow 0$, and also as $\xi \rightarrow \pi$ since $m>0$.

In Figures 11(a) and (b) a $\mu=1$ and 2 (outlet: $M=0 \cdot 3, h=0 \cdot 6$ ), and in Figure 12 a $\mu=1(M=0 \cdot 3, h=0.95)$ wave radiating from an outlet is seen, in each case with and, for comparison, without a Kutta condition. The Kutta condition is clearly seen to decrease the amplitude in all directions except for that of the main lobe, which is left unchanged. Primarily, the diffracted field in the shadow zone is affected. It is noted that this decrease is further amplified for higher Mach numbers. The power loss $10_{10} \log \left(P^{f} / P^{t}\right)$ in these cases amounts to $-0.096 \mathrm{~dB}(\mu=1, h=0 \cdot 6),-0.104 \mathrm{~dB}(\mu=2, h=0 \cdot 6)$, and $-0.426 \mathrm{~dB}$ $(\mu=1, h=0 \cdot 95)$; so here, in contrast to the behaviour for the lower values of $k$ discussed previously, the effect of vortex shedding acoustically is not so much found in the net 

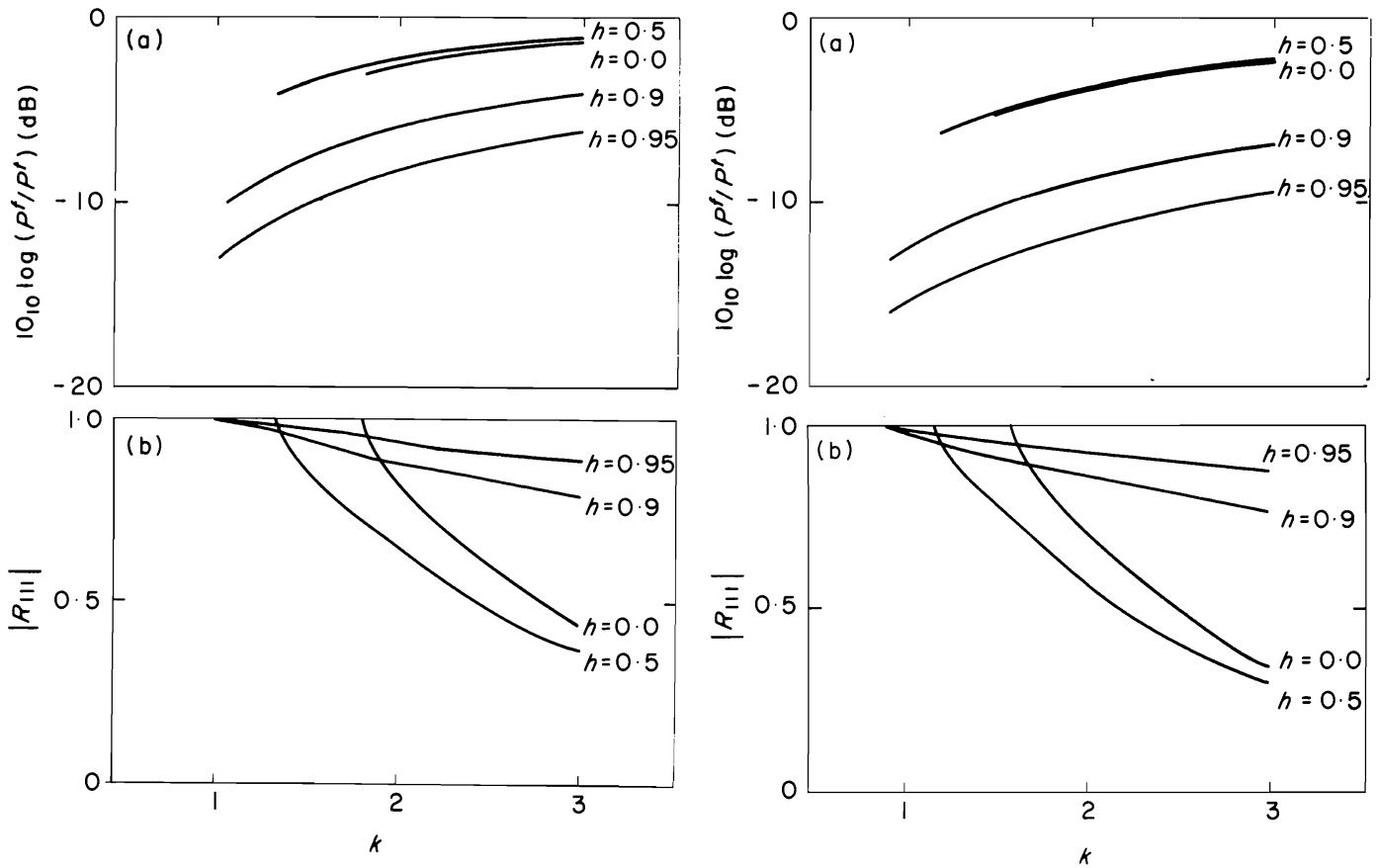

Figure 7. (a) Power loss of a $m=1, \mu=1$ wave for $M=0.2$, hub radii $h=0,0.5,0.9,0.95$, and $k$ between the first cut-off frequency and 3 ; (b)

Figure 8. The same as Figures $7(\mathrm{a})$ and (b) but modulus of reflection coefficient $\left|R_{111}\right|$ for same conwith $M=0.5$. ditions as (a).

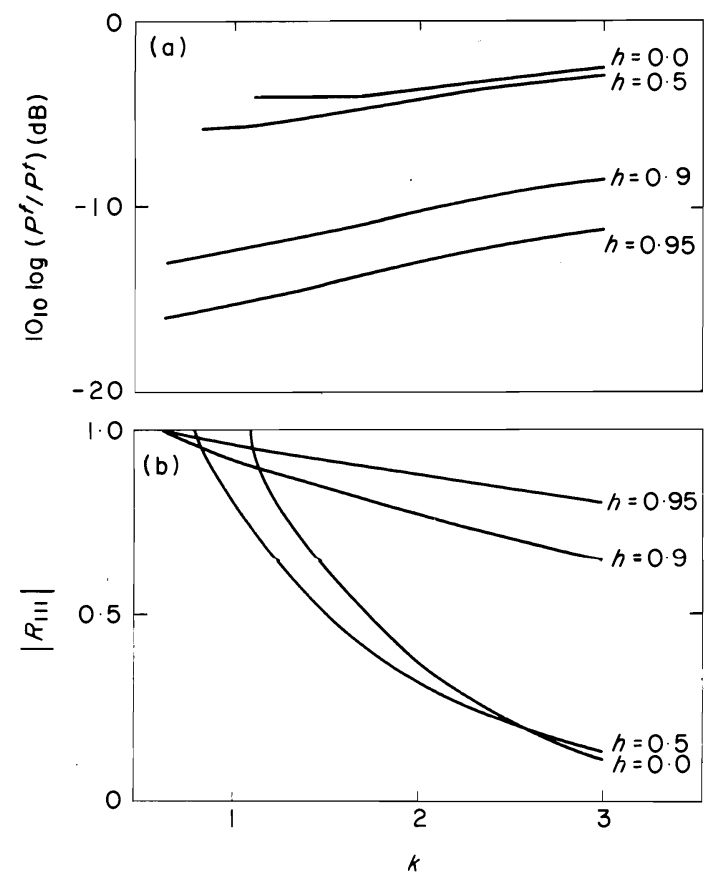

Figure 9. The same as Figures 7 (a) and (b) but with $M=0 \cdot 8$. 


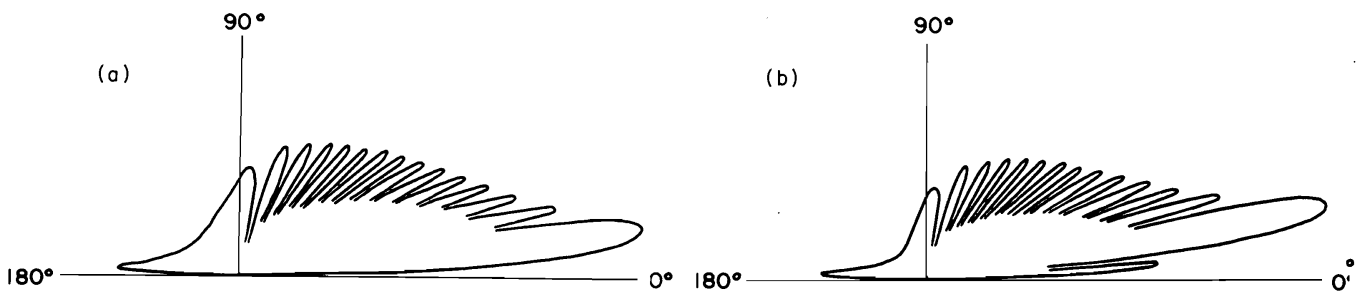

Figure 10. (a) Radiation pattern for $k=50, m=4, M=-0 \cdot 3$ (inlet), $h=0, \mu=1, \gamma=0$; (b) the same as (a) but with $\mu=2$.
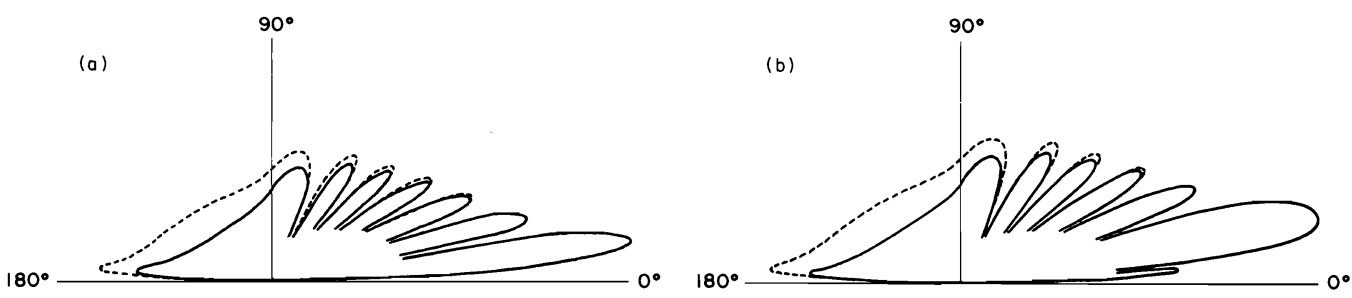

Figure 11. (a) Radiation pattern for $k=50, m=4, M=0 \cdot 3$ (outlet), $h=0 \cdot 6, \mu=1,-, \gamma=1 ; \cdots \cdots$, $\gamma=0$; (b) the same as (a) but with $\mu=2$.

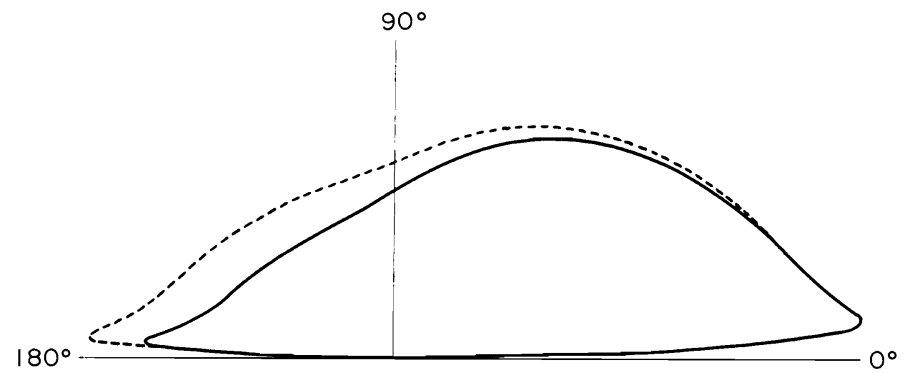

Figure 12. The same as Figure $11(\mathrm{a})$ but with $h=0.95$.

absorption of energy but mainly in the radiation pattern. The effect of the hub is twofold: the number of cut-on modes in the pipe decreases and with it the number of lobes in the far field, while at the same time the shadow field is amplified. Apart from the zeros at $\xi=0$ and $\xi=\pi$, the far field tends to be omnidirectional as $h \rightarrow 1$.

\section{CONCLUDING REMARKS}

A comprehensive analytical solution has been derived, by using the Wiener-Hopf technique, of the problem of sound propagation in and radiation from a semi-infinite cylindrical pipe with a doubly infinite hub, submerged in a subsonic coaxial uniform mean flow. For completeness, the limiting forms of zero Mach number and zero hub radius have been included. Particular attention has been paid to the unsteady shedding of vorticity, in the case of an outflow, caused by the acoustic wave scattering at the pipe edge. Since with an inflow no vorticity is shed, and with an outflow the amount of shed vorticity is not always exactly known, a complex parameter $\gamma$ is introduced. This parameter 
controls the rate of vortex shedding in a way that $\gamma=0$ relates to no vorticity, and $\gamma=1$ to a finite velocity near the edge (Kutta condition). Mathematically, the field of the vortex sheet is an eigensolution of the equations, coming into play after removing the condition of continuity along the cylindrical surface formed by the extension of the pipe. The amplitude of this eigensolution is just the factor $\gamma$. This vorticity field appears as an extra pole of the spatial Fourier transform.

The final solution is given as a Fourier integral, which is still difficult to handle since no explicit form is available. In fact it is a double integral, since a function $\tilde{K}_{-}$, part of the integrand, is, in turn, also defined by an integral. However, in some of the physically interesting parts of the field, the Fourier integral can be evaluated, greatly simplifying the formulae and leaving only the evaluation of $\tilde{K}_{-}$. These more explicit representations of the solution are the modal series inside the pipe, the acoustic far field propagating radially away from the exit, and the hydrodynamic far field confined to the immediate neighbourhood of the vortex sheet.

At a certain frequency the axial wave length of a modal wave form inside the pipe tends to infinity, and the wave is in resonance. Below this frequency the wave does not propagate (i.e., is cut off). To complete the description of the field the behaviour at this cut-off frequency has been analyzed in some detail.

To evaluate the field numerically, it is necessary to compute the integral defining $\tilde{K}_{-}$. A method for this, different from that usually presented, has been described and analyzed, and involves the following steps: the interval of integration $[0, \infty)$ is divided into $[0, A]$ and $[A, \infty)$ where $A$ is chosen just large enough to permit an asymptotic evaluation along $[A, \infty)$, while the contour along $[0, A]$ is deformed into a parabola in the complex plane to circumvent the singularities of the integrand on the real interval. The price paid is the computation of complex Bessel functions, but the gain in simplicity makes the method more than worthwhile, especially at higher frequencies.

A particularly interesting aspect of the field is the energy balance. Therefore the power, a quantity defined as the time averaged energy flux (= intensity) integrated over a given surface, has been considered. If one chooses the surfaces properly, one can calculate various powers explicitly. Since when energy is conserved the power flow across a closed surface equals zero, one then has available, on the one hand, a convenient check on the numerical calculations, and, on the other hand, a detailed quantification of the acoustic energy loss, known to occur due to the vortex shedding. Explicit formulae have been obtained for the power of the field on a cross section in the pipe, of the acoustic far field, of the hydrodynamic far field, and the power difference across two cylindrical surfaces enclosing the vortex sheet. This fully describes the energy balance, since the acoustic far field power, the hydrodynamic far field power and the power absorbed by the wake add up to the power of the field in the pipe. Furthermore, this non-zero power absorption illustrates that vorticity may act as a source, which indeed precludes any definition of acoustic energy (in terms of only the acoustic variables) in a rotational flow region. This unfortunate consequence of the linearization has an important implication for the necessary boundary conditions of the present problem: near the vortex sheet the radiation condition, separating incoming and outgoing waves at infinity, can no longer be defined, at least not via the energy flux vector; instead, arguments of causality can be introduced, but they may not necessarily be equivalent to energy considerations. See also the discussion in reference [20].

The well-known high acoustic power loss of sound transmitted through a jet for low Helmholtz numbers $[17,18]$ raised the questions (i) whether or not the same is true in uniform (out-)flow, (ii) whether it is primarily related to the pipe diameter rather than to the effective surface of the cross section, and (iii) whether or not it is a property of 
any mode at its cut-off frequency. In an attempt to answer these questions some examples were computed numerically. It was found that (i) for a low Helmholtz number plane wave the attenuation in uniform flow is of the same order of magnitude (in fact even higher), increasing with the Mach number, and also increasing with the hub diameter so that (ii) a cross-sectional surface is indeed the relevant parameter. Down to the cut-off frequencies of other modes the attenuation tends to a maximum, but a finite maximum, so that (iii) the effect (in uniform flow) is not specifically inherent to cut-off. In addition to these low frequency calculations, a typical high frequency $(k=50)$ example was studied. Here the effect of vortex shedding is not so much found in the acoustic power loss but rather in the far field radiation pattern, where, in general, it results in a decrease in all directions except near the main lobe. Variation of Mach number or hub radius affects mainly the number of cut-on modes, and thereby the number of lobes in the far field.

\section{ACKNOWLEDGMENTS}

The present study was partially sponsored by the Netherlands Agency for Aerospace Programs (NIVR). The author acknowledges the programming of the complex Bessel function computer routines by $\mathrm{Mr}$ J. H. Meelker, and the programming of the sound radiation and reflection problem by $\mathrm{Mr} \mathrm{A}$. L. Bleekrode and $\mathrm{Mr} \mathrm{C}$. H. Hofstra.

\section{REFERENCES}

1. LORD RAYLEIGH 1945 The Theory of Sound. New York: Dover, second edition.

2. H. LEVINE and J. SCHWINGER 1948 Physical Review 73, 383-406. On the radiation of sound from an unflanged circular pipe.

3. G. F. CARRIER 1956 Quarterly of Applied Mathematics 13, 457-461. Sound transmission from a tube with flow.

4. R. M. MUNT 1975 Journal of the Institute of Mathematics and Applications 16, 1-10. Acoustic radiation from a circular cylinder in a subsonic stream.

5. D. G. CRIGHTON 1972 Journal of Fluid Mechanics 51, 357-362. Radiation from vortex filament motion near a half plane.

6. N. FRIEDRICH $1975 D L R-F B$ 75-24. Untersuchungen zur Schallabstrahlung aus einem in axialer Richtung angeströmten Ringkanal.

7. R. M. MUNT 1977 Journal of Fluid Mechanics 83, 609-640. The interaction of sound with a subsonic jet issuing from a semi-infinite cylindrical pipe.

8. G. N. WATSON 1966 A Treatise on the Theory of Bessel Functions. Cambridge University Press, second edition.

9. M. E. GoldSTEIN 1976 Aeroacoustics. New York: McGraw-Hill.

10. B. NOBLE 1958 Methods Based on the Wiener-Hopf Technique for the Solution of Partial Differential Equations. London: Pergamon Press.

11. S. W. RIENSTRA 1981 Journal of Fluid Mechanics 108, 443-460. Sound diffraction at a trailing edge.

12. C. L. MORfEY 1971 Journal of Sound and Vibration. 14, 37-55. Sound transmission and generation in ducts with flow.

13. D. S. JONES 1955 Philosophical Transactions of the Royal Society A247, 499-528. The scattering of a scalar wave by a semi-infinite rod of circular cross section.

14. L. A. WEINSTEIN 1969 The Theory of Diffraction and the Factorization Method. Boulder, Colorado: Golem Press.

15. A. ERdélyi, W. MAgnus, F. OBerhetTinger and F. G. Tricomi 1953 Higher Transcendental Functions Volume II. New York: McGraw-Hill.

16. R. RAMAKRISHNAN 1982 Journal of Sound and Vibration 81, 592-595. A note on the calculation of Wiener-Hopf split functions.

17. D. Bechert, U. Michel and E. Pfizenmaier 1977. American Institute of Aeronautics and Astronautics Paper No. 77-1278. Experiments on the transmission of sound through jets.

18. M. S. HOWE 1979 Journal of Fluid Mechanics 91, 209-229. Attenuation of sound in a low Mach number nozzle flow. 
19. A. M. CARgill 1979 in Mechanics of Sound Generation in Flows (editor E.-A. Müller), Berlin: Springer-Verlag. Low frequency sound radiation due to the interaction of unsteady flows with a jet pipe.

20. S. W. RIENSTRA 1983 Journal of Sound and Vibration 86, 539-556. A small Strouhal number analysis for acoustic wave-jet flow-pipe interaction.

21. M. ABRAMOWITZ and I. A. STEGUN 1964 Handbook of Mathematical Functions. National Bureau of Standards.

APPENDIX: THE ASYMPTOTIC APPROXIMATION OF $\frac{\mathrm{i} t}{\pi} \int_{A}^{\infty} \frac{\log \tilde{K}(\tau)}{\tau^{2}-t^{2}} \mathrm{~d} \tau$ FOR A LARGE

If $\tau$ is along the contour, $1<A \leqslant \tau<\infty$, then $\arg v(\tau)=-\frac{1}{2} \pi$, so if $k v(\tau) / \beta=-\mathrm{i} y, y$ is real. Then

$$
L(-\mathrm{i} y)=2\left[\mathrm{~K}_{m}^{\prime}(y) / \mathrm{K}_{m}^{\prime}(y h)\right]\left\{\mathrm{I}_{m}^{\prime}(y h) \mathrm{K}_{m}^{\prime}(y)-\mathrm{I}_{m}^{\prime}(y) \mathrm{K}_{m}^{\prime}(y h)\right\},
$$

with $\mathrm{I}_{m}$ and $\mathrm{K}_{m}$ modified Bessel functions of the first and second kind, respectively [8]. From Abramowitz and Stegun [21, p. 378], it follows that

so that

$$
L(-\mathrm{i} y) \cong \frac{1}{y}\left\{1+\frac{l_{2}}{y^{2}}+\frac{l_{4}+\frac{1}{2} l_{2}^{2}}{y^{4}}+\cdots\right\} \quad(y \rightarrow \infty, h \text { fixed })
$$

$$
[\log L(-\mathrm{i} y)]_{a s}=-\log y+\frac{l_{2}}{y^{2}}+\frac{l_{4}}{y^{4}},
$$

with $l_{2}=\frac{1}{8}\left(4 m^{2}-3\right)$ and $l_{4}=-\frac{1}{64}\left(16 m^{4}-104 m^{2}+27\right)$. Hence, after some algebra, one obtains, for sufficiently large $k v(A) / \beta$ with $A>1$, and $h$ fixed,

$$
\begin{aligned}
\frac{\mathrm{i} t}{\pi} \int_{A}^{\infty} \frac{[\log L(k v(\tau) / \beta)]_{a s}}{\tau^{2}-t^{2}} \mathrm{~d} \tau & =\frac{1}{2 \pi \mathrm{i}}\left\{\left(\log (k / \beta)+\tilde{l}_{2}-\tilde{l}_{4}+\frac{1}{2} \log \left(A^{2}-t^{2}\right)\right) \log \frac{A+t}{A-t}\right. \\
& +\frac{1}{2} \sum_{n=1}^{\infty} \frac{1}{n^{2}}\left[\left(\frac{t-1}{t+A}\right)^{n}-\left(\frac{t-1}{t-A}\right)^{n}+\left(\frac{t+1}{t+A}\right)^{n}-\left(\frac{t+1}{t-A}\right)^{n}\right] \\
& \left.-t\left(\tilde{l}_{2}-\frac{1}{2}\left(3-t^{2}\right) \tilde{l}_{4}\right) \log \frac{A+1}{A-1}-t\left(1-t^{2}\right) \tilde{l}_{4} \frac{A}{A^{2}-1}\right\},
\end{aligned}
$$

with $\tilde{l}_{n}=l_{n} /(k / \beta)^{n}\left(1-t^{2}\right)^{n / 2}(n=2,4)$ and where for the logarithms principal branches are to be taken. If $t$ is real and $A<2 t+1$ the series does not converge, since then $|(t+1) /(t-A)|>1$. In this case the series should be slightly rewritten, in such a way that $-\sum_{n=1}^{\infty}\left(1 / n^{2}\right)[(t+1) /(t-A)]^{n}$ becomes

$$
\frac{\pi^{2}}{6}+\frac{1}{2} \log ^{2}\left(\frac{t+1}{A-t}\right)+\sum_{n=1}^{\infty} \frac{1}{n^{2}}\left(\frac{t-A}{t+1}\right)^{n} .
$$

If $t=1$ one has to use the limiting value of the above expression for the integral, which becomes

$$
\begin{aligned}
\frac{1}{2 \pi \mathrm{i}} & \left\{\left(\log (k / \beta)+\frac{1}{2} \frac{l_{2}}{(k / \beta)^{2}}-\frac{3}{8} \frac{l_{4}}{(k / \beta)^{4}}+\frac{1}{2} \log \left(A^{2}-1\right)\right) \log \frac{A+1}{A-1}\right. \\
& -\left(\frac{l_{2}}{(k / \beta)^{2}}-\frac{3}{4} \frac{l_{4}}{(k / \beta)^{4}}\right) \frac{A}{A^{2}-1}-\frac{1}{2} \frac{l_{4}}{(k / \beta)^{4}} \frac{A}{\left(A^{2}-1\right)^{2}} \\
& \left.+\frac{1}{2} \sum_{n=1}^{\infty} \frac{1}{n^{2}}\left[\left(\frac{2}{A+1}\right)^{n}-\left(\frac{2}{1-A}\right)^{n}\right]\right\},
\end{aligned}
$$

with the appropriately adapted form of the series if $A<3$. 